\begin{tabular}{|c|l|}
\hline Title & A Rohlin property for one parameter automorphism groups \\
\hline Author(s) & Kishimoto, A. \\
\hline Citation & Hokkaido University Preprint Series in Mathematics, 296, 1-27 \\
\hline Issue Date & 1995-6-1 \\
\hline DOI & 10.14943/83443 \\
\hline Doc URL & http://hdl.handle.net/2115/69047 \\
\hline Type & bulletin (article) \\
\hline File Information & pre296.pdf \\
\hline
\end{tabular}

Instructions for use 
A Rohlin property for one-parameter automorphism groups

\author{
Akitaka Kishimoto
}

Series $\sharp 296$. June 1995 


\section{HOKKAIDO UNIVERSITY \\ PREPRINT SERIES IN MATHEMATICS}

\#272 S. Izumiya, A. Takiyama, A time-like surface in Minkowski 3-space which contains light-like lines, 7 pages. 1994.

\#273 K. Tsutaya, Global existence of small amplitude solutions for the Klein-Gordon-Zakharov equations, 11 pages. 1994.

$\sharp 274$ H. Kubo, On the critical decay and power for semilinear wave equations in odd space dimensions, 22 pages. 1994.

$\sharp 275$ N. Terai, T. Hibi, Alexander duality theorem and second Betti numbers of Stanley-Reisner rings, 2 pages. 1995.

$\sharp 276$ N. Terai, T. Hibi, Stanley-Reisner rings whose Betti numbers are independent of the base field, 12 pages. 1995.

$\sharp 277$ N. Terai, T. Hibi, Computation of Betti numbers of monomial ideals associated with cyclic polytopes, 11 pages. 1995.

$\sharp 278$ N. Terai, T. Hibi, Computation of Betti numbers of monomial ideals associated with stacked polytopes, 8 pages. 1995.

$\sharp 279$ N. Terai, T. Hibi, Finite free resolutions and 1-skeletons of simplicial $(d-1)$-spheres, 3 pages. 1995.

$\sharp 280$ N. Terai, T. Hibi, Monomial ideals and minimal non-faces of Cohen-Macaulay complexes, 6 pages. 1995.

$\sharp 281$ A. Arai, N. Tominaga, Analysis of a family of strongly commuting self-adjoint operators with applications to perturbed d'Alembertians and the external field problem in quantum field theory, 44 pages. 1995.

\#282 T. Mikami, Asymptotic behavior of the first exit time of randomly perturbed dynamical systems with a repulsive equilibrium point, 29 pages. 1995.

$\sharp 283$ K. Iwata, J. Schäfer, Markov property and cokernels of local operators, 17 pages. 1995.

$\sharp 284$ T. Nakazi, M. Yamada, Riesz's Functions In Weighted Hardy And Bergman Spaces, 20 pages. 1995.

$\sharp 285$ K. Hidano, K. Tsutaya, Scattering theory for nonlinear wave equations in the invariant Sobolev space, 32 pages. ' 1995.

\#286. A. Arai, Strong coupling limit of the zero-energy-state density of the Dirac-Weyl operator with a singular vector potential, 8 pages. 1995.

$\# 287$ T. Nakazi, Factorizations of outer functions and extremal problems, 15 pages. 1995.

$\sharp 288$ A. Kishimoto, The Rohlin property for automorphisms of UHF algebras, 15 pages. 1995.

\#289 K. Goto, A. Yamaguchi and I. Tsuda, Nine-bit states cellular automata are capable of simulating the pattern dynamics of coupled map lattice, 24 pages. 1995.

$\sharp 290$ Y. Giga, Interior derivative blow-up for quasilinear parabolic equations, 16 pages. 1995.

$\sharp 291$ F. Hiroshima, Functional Integral Representation of a Model in QED, 48 pages. 1995.

\# 292 N. Kawazumi, A Generalization of the Morita-Mumford Classes to Extended Mapping Class Groups for Surfaces, 11 pages. 1995.

\#293 P. Aviles and Y. Giga, The distance function and defect energy, 23 pages. 1995.

\#294 S. Izumiya and A. Takiyama, A time-like surface in Minkowski 3-space which contains pseudocircles, 11 pages. 1995.

\# 295 S. Izumiya, Local classifications of multi-valued solutions of quasilinear first order partial differential equations, 12 pages. 1995. 


\title{
A Rohlin property for one-parameter automorphism groups
}

\author{
Akitaka Kishimoto \\ Department of Mathematics, Hokkaido University \\ Sapporo 060 Japan
}

Dedicated to Professor H. Hasegawa

June 1995

\begin{abstract}
We define a Rohlin property for one-parameter automorphism groups of unital simple $C^{*}$-algebras and show that for such an automorphism group any cocycle is almost a coboundary. We apply the same method to the single automorphism case and show that if an automorphism of a unital simple $C^{*}$-algebra with a certain condition has a central sequence of approximate eigen-unitaries for any complex number of modulus one, then any cocycle is almost a coboundary, or the automorphism has the stability. We also show that if a one-parameter automorphism group of a unital separable purely infinite simple $C^{*}$-algebra has the Rohlin property then the crossed product is simple and purely infinite.
\end{abstract}

\section{Introduction}

A $C^{*}$-dynamical system is a $C^{*}$-algebra with an action of a locally compact group by automorphisms. To analyse such a system the notion of Rohlin property was introduced and exploited at least when the group is the integer group $\mathbf{Z}$ or perhaps an amenable discrete group $[8,12,13,5,3,4,22,26,19,20]$. We here introduce a Rohlin property for one-parameter automorphism groups; if $\alpha$ is a strongly continuous one-parameter automorphism group of a unital simple $C^{*}$-algebra $A, \alpha$ is said to have the Rohlin property if for any real number $p \in \mathbf{R}$ there is a central sequence $\left\{v_{n}\right\}$ of unitaries in $A$ such that $\alpha_{t}\left(v_{n}\right)-e^{i p t} v_{n}$ converges to zero uniformly in $t$ on every bounded subset of $\mathbf{R}$. In this case the spectral projections of $v_{n}$ would be periodically transformed, in a sense, under $\alpha_{t}$ with period $2 \pi / p$; so this is an analogue of the Rohlin property for single automorphisms. The main result (Theorem 2.1 ) will show that then for any $\alpha$-cocycle $u$, i.e., a continuous family $u(t)$ of unitaries with $u(s) \alpha_{s}(u(t))=u(s+t), s, t \in \mathbf{R}$ is almost a coboundary, i.e., 
has a sequence $\left\{w_{n}\right\}$ of unitaries with $w_{n} \alpha_{t}\left(w_{n}^{*}\right) \rightarrow u(t)$ uniformly in $t$ on every bounded subset of $\mathbf{R}$. (Here a small condition on $u$ should be imposed; see 2.1 for details.) The only natural examples we can give of one-parameter automorphism groups with the Rohlin property are on simple non-commutative tori (Proposition 2.5). (Others may be obtained by considering infinite tensor products.) Note that any one-parameter automorphism group of an AF algebra does not have the Rohlin property since $K_{1}$ is trivial (the unitaries $\left\{v_{n}\right\}$ above must give non-trivial elements in $K_{1}$ ). This is very much different from the situation for single automorphisms [20]. We also note that the crossed product by a oneparameter automorphism group with the Rohlin property must be simple (Proposition 2.4).

We employ a similar method to show that if an automorphism $\alpha$ of a unital simple $C^{*}$-algebra with a certain condition (which is satisfied by AF algebras and by purely infinite $C^{*}$-algebras if $\alpha_{*}=i d$ on $K_{0}$ ), has the property that for any complex number $\lambda$ of modulus one there is a central sequence $\left\{v_{n}\right\}$ of unitaries such that $\alpha\left(v_{n}\right)-\lambda v_{n} \rightarrow 0$, then for any unitary $u$ in the connected component of 1 of the unitary group of $A$ there is a sequence $\left\{w_{n}\right\}$ of unitaries such that $w_{n} \alpha\left(w_{n}^{*}\right) \rightarrow u$ (Theorem 3.2). If the $C^{*}$-algebra is AF and the automorphism induces the trivial action on $K_{0}$, the above property is equivalent to the Rohlin property. (This is perhaps not surprising because the Rohlin property may be obtained by the property that any non-zero power of the automorphism is not weakly inner in any tracial representation [20], which follows easily from the above property.)

If a one-parameter automorphism group $\alpha$ has the Rohlin property then there are $\alpha$ covariant irreducible representations [17]. If the $C^{*}$-algebra has real rank zero and satisfies the condition referred to above, we shall show that there is a decreasing sequence of almost $\alpha$-invariant projections whose limit is a minimal projection in the second dual (Theorem 4.1). (A similar result for single automorphisms with trivial action on $K_{0}$ can be obtained by using part of the arguments for 4.1 if the automorphism satisfies the property that all non-zero powers are outer; a substantially weaker property than the Rohlin property in general.) We have an example of one-parameter automorphism groups where the conclusion of Theorem 4.1 does not hold. (In this example $\alpha_{1}$ is inner.) Then we shall show that the crossed product of a unital separable purely infinite simple $C^{*}$-algebra by a one-parameter automorphism group with the Rohlin property is simple and purely infinite (Theorem 4.8).

\section{One-parameter automorphism groups}

Let $A$ be a unital $C^{*}$-algebra and let

$$
A^{\infty}=l^{\infty}(\mathbf{N}, A) / c_{0}(\mathbf{N}, A)
$$

which is a unital $C^{*}$-algebra; $x=\left(x_{n}\right)+c_{0}(\mathbf{N}, A)$ has norm limsup $\left\|x_{n}\right\|$. Embedding $A$ into $l^{\infty}(\mathbf{N}, A)$ by $x \rightarrow(x, x, \ldots)$ and also into $A^{\infty}$, we denote $A^{\infty} \cap A^{\prime}$ by $A_{\infty}$. Let $\alpha$ be a strongly continuous one-parameter group of automorphisms of $A$; then $\alpha$ acts on 
$l^{\infty}(\mathbf{N}, A)$ in the natural way and leaves $c_{0}(\mathbf{N}, A)$ and $A$ invariant. Let

$$
l_{\alpha}^{\infty}(\mathbf{N}, A)=\left\{x \in l^{\infty}(\mathbf{N}, A) ; t \rightarrow \alpha_{t}(x) \text { is continuous }\right\}
$$

which is a $C^{*}$-subalgebra of $l^{\infty}(\mathbf{N}, A)$ containing $c_{0}(\mathbf{N}, A)$ and $A$, and let

$$
A_{\alpha}^{\infty}=l_{\alpha}^{\infty}(\mathbf{N}, A) / c_{0}(\mathbf{N}, A), \quad A_{\infty, \alpha}=A_{\alpha}^{\infty} \cap A^{\prime} .
$$

By an $\alpha$-cocycle $u$ in $A_{\infty, \alpha}$ we mean a continuous family $u(t), t \in \mathbf{R}$ of unitaries in $A_{\infty, \alpha}$ such that for $s, t \in \mathbf{R}$

$$
u(s) \alpha_{s}(u(t))=u(s+t) .
$$

For a unitary $u \in A$, if $u$ is in the connected component of 1 of the unitary group of $A$, let $l(u)$ be the infimum of the lengths of rectifiable paths from $u$ to 1 and otherwise let $l(u)=\infty$. If $A$ has real rank zero, then either $l(u) \leq \pi$ or $l(u)=\infty$ [24].

Theorem 2.1 Let $A$ be a unital separable simple $C^{*}$-algebra and let $\alpha$ be a strongly continuous one-parameter automorphism group of $A$. Then the following conditions are equivalent:

1. For each $p \in \mathbf{R}$ there exists a unitary $v \in A_{\infty, \alpha}$ such that $\alpha_{t}(v)=e^{i t p} v$.

2. For each $\alpha$-cocycle $u$ in $A_{\infty, \alpha}$ such that $t^{-1} l(u(t)) \rightarrow 0$ as $t \rightarrow 0$, there exists a unitary $w \in A_{\infty, \alpha}$ such that $u(t)=w \alpha_{t}\left(w^{*}\right)$.

In this case for each $\alpha$-cocycle $u$ in $A$ such that $t^{-1} l(u(t)) \rightarrow 0$ as $t \rightarrow 0$, there is a sequence $\left\{w_{n}\right\}$ of unitaries in $A$ such that $\left\|u(t)-w_{n} \alpha\left(w_{n}^{*}\right)\right\| \rightarrow 0$ uniformly in $t$ on each compact subset of $\mathbf{R}$.

A one-parameter automorphism group $\alpha$ of a unital simple $C^{*}$-algebra $A$ is said to have the Rohlin property if $\alpha$ satisfies the condition (1) in the above theorem.

Let $T$ be a subset of $A$. We say that $T$ is equicontinuous with respect to $\alpha$ if the family of continuous functions $t \rightarrow \alpha_{t}(x)$ with $x \in T$ is equicontinuous, i.e., for any $\epsilon>0$ there exists a $\delta>0$ such that if $|t|<\delta$ and $x \in T$, then $\left\|\alpha_{t}(x)-x\right\|<\epsilon$.

Lemma 2.2 Let $u(t), t \in \mathbf{R}$ be a continuous family of unitaries in $A_{\infty, \alpha}$. Then there exists a sequence $\left\{u_{n}(t)\right\}$ of continuous families of unitaries in $A$ such that $\left(u_{1}(t), u_{2}(t), \ldots\right)$ represents $u(t),\left\{u_{n}(\cdot)\right\}$ is equicontinuous on every compact subset of $\mathbf{R}$ and $\left\{u_{n}(t)\right\}$ is equicontinuous with respect to $\alpha$ for each $t \in \mathbf{R}$. Moreover if $u$ satisfies that $\|u(s)-u(t)\|<$ $C|s-t|$ for distinct $s, t \in[-r, r]$ with some $C, r>0$ then $\left\{u_{n}\right\}$ can be chosen so that $\left\|u_{n}(s)-u_{n}(t)\right\|<C|s-t|$ for distinct $s, t \in[-r, r]$, for all sufficiently large $n$.

Proof. For each $t \in \mathbf{R}$ there is a sequence $\left\{x_{n}\right\}$ in $A$ representing $u(t)$ such that $x_{n}$ 's are equicontinuous with respect to $\alpha$. Since $x_{n}^{*} x_{n} \rightarrow 1$ and $x_{n} x_{n}^{*} \rightarrow 1, u_{n}=x_{n}\left|x_{n}\right|^{-1}$ is welldefined for large $n$ and $\left\{u_{n}\right\}$ satisfies the same properties as $\left\{x_{n}\right\}$. Thus we can assume that $x_{n}$ 's are unitaries. 
For each $t \in \mathbf{R}$ let $\left\{\tilde{u}_{n}(t)\right\}$ be a sequence of unitaries in $A$ representing $u(t)$ such that $\tilde{u}_{n}(t)$ 's are equicontinuous with respect to $\alpha$. Let $n_{0}=0$. For each $k \in \mathbf{N}$ we choose an $n_{k} \in \mathbf{N}$ such that $n_{k}>n_{k-1}$ and if $s, t \in[-k, k]$ satisfies $|s-t|<2^{-n_{k}}$ then $\|u(s)-u(t)\|<1 / 3 k$. Let $m_{0}=0$. Then we choose an $m_{k} \in \mathbf{N}$ such that $m_{k}>m_{k-1}$ and for any $l \geq m_{k}$ and $s, t \in P_{k} \equiv\left\{j \in[-k, k] \mid 2^{n_{k}} j \in \mathbf{Z}\right\}$

$$
\left\|\tilde{u}_{l}(s)-\tilde{u}_{l}(t)\right\|<\|u(s)-u(t)\|+1 / 3 k .
$$

For $l$ with $m_{k} \leq l<m_{k+1}$ and $s \in P_{k}$ define $h_{l, s}=h_{l, s}^{*} \in A$ of small norm by

$$
\tilde{u}_{l}\left(s+2^{-n_{k}}\right)=e^{i h_{l, s}} \tilde{u}_{l}(s) .
$$

Let

$$
u_{l}(t)=e^{i(t-s) 2^{n_{k} h_{l, s}}} \tilde{u}_{l}(s), t \in\left[s, s+2^{-n_{k}}\right],
$$

$u_{l}(t)=\tilde{u}_{l}(-k), t \leq-k$, and $u_{l}(t)=\tilde{u}_{l}(k), t \geq k$. Thus we obtain the continuous functions $u_{n}$ on $\mathbf{R}$ for $n \geq m_{1}$. We assert that $\left\{u_{n}\right\}$ satisfies the required properties.

For $t \in 2^{-m} \mathbf{Z}$ with $m \in \mathbf{N},\left\{u_{n}(t)\right\}$ represents $u(t)$. Let $s, t \in \mathbf{R}$. For a sufficiently large $k$ with $s, t \in[-k, k]$ we have that for $n \geq m_{k}$,

$$
\begin{aligned}
\left\|u_{n}(s)-u_{n}(t)\right\| & \leq\left\|u_{n}(s)-u_{n}\left(s^{\prime}\right)\right\|+\left\|u_{n}\left(s^{\prime}\right)-u_{n}\left(t^{\prime}\right)\right\|+\left\|u_{n}\left(t^{\prime}\right)-u_{n}(t)\right\| \\
& <\|u(s)-u(t)\|+1 / k
\end{aligned}
$$

where $s^{\prime}, t^{\prime} \in P_{k}$ with $\left|s-s^{\prime}\right|<2^{-n_{k}},\left|t-t^{\prime}\right|<2^{-n_{k}}$. Hence $\left\{u_{n}(\cdot)\right\}$ is equicontinuous on each bounded interval of $\mathbf{R}$. In particular $\left\{u_{n}(t)\right\}$ represents $u(t)$ for each $t \in \mathbf{R}$.

If $s \in 2^{-m} \mathbf{Z}$ with $m \in \mathbf{N}$, then $\left\{u_{n}(s)\right\}$ is equicontinuous with respect to $\alpha$. Since $\left\{u_{n}(\cdot)\right\}$ is equicontinuous, this is the case for any $t \in \mathbf{R}$.

To show the last assertion choose an increasing sequence $\left\{n_{k}\right\}$ such that for $n \in$ $\left\{n_{k}, n_{k}+1, \ldots, n_{k+1}-1\right\}$ and $l=-2^{k}+1,-2^{k}+2, \ldots, 2^{k}$,

$$
\epsilon_{n} \equiv\left\|u_{n}\left(r 2^{-k}(l-1)\right)-u_{n}\left(r 2^{-k} l\right)\right\|<C r 2^{-k} .
$$

Suppose that $C r 2^{-k}<2$. We define $u_{n}^{\prime}$ by: For $t \in\left[r 2^{-k}(l-1), r 2^{-k} l\right]$,

$$
u_{n}^{\prime}(t)=e^{i\left(2^{k} t-r(l-1)\right) / r \cdot h} u_{n}\left(r 2^{-k}(l-1)\right)
$$

where $h$ is defined as before by $\log \left(u_{n}\left(r 2^{-k} l\right) u_{n}\left(r 2^{-k}(l-1)\right)^{*}\right)$ with branch along the negative real axis. Then since $\|h\| \leq 2 \arcsin \epsilon_{n} / 2$, the new $\left\{u_{n}^{\prime}\right\}$ satisfies the required properties.

Lemma 2.3 Let $A$ be a unital simple $C^{*}$-algebra and let $\left\{v_{n}\right\}$ be a central sequence of unitaries in $A$ such that for any $\epsilon>0, \operatorname{Sp}\left(v_{n}\right)+(0, \epsilon)=\mathbf{T}$ (regarded as $\mathbf{R} / \mathbf{Z}$ ) for all sufficiently large $n$. Define a linear map of the algebraic tensor product $A \odot C(\mathbf{T})$ into $A$ by

$$
\phi_{n}(a \otimes f)=a f\left(v_{n}\right)
$$


Then $\left\{\phi_{n}\right\}$ is an approximate homomorphism, i.e.,

$$
\left\|\phi_{n}(x)^{*}-\phi_{n}\left(x^{*}\right)\right\|,\left\|\phi_{n}(x y)-\phi_{n}(x) \phi_{n}(y)\right\|
$$

converge to zero for any $x, y \in A \odot C(\mathbf{T})$, and for any $x \in A \odot C(\mathbf{T})$,

$$
\lim \left\|\phi_{n}(x)\right\|=\|x\|
$$

where $\|x\|$ is the $C^{*}$-norm of $x \in A \otimes C(\mathbf{T})$.

Proof. It follows by easy computations that $\left\{\phi_{n}\right\}$ is an approximate homomorphism. Then the map $x \mapsto\left(\phi_{1}(x), \phi_{2}(x), \ldots\right)$ defines a homomorphism of $A \odot C(\mathbf{T})$ into $A^{\infty}$ and so

$$
\gamma(x)=\lim \sup \left\|\phi_{n}(x)\right\|
$$

defines a $C^{*}$-seminorm on $A \odot C(\mathbf{T})$. (See $[23,9]$ for similar arguments.)

Let $I$ be an open interval in $\mathbf{T}$ and let for $a \in A$

$$
\delta(a)=\sup \left\{\gamma(a \otimes f) \mid 0 \leq f \leq \chi_{I}\right\}
$$

where $\chi_{I}$ is the characteristic function of $I$. Then it follows that $\delta$ is a $C^{*}$-seminorm on $A$. Since $\delta(1)=1$ by the assumption on $\operatorname{Sp}\left(v_{n}\right)$ and since $A$ is simple, it follows that $\delta(a)=\|a\|, a \in A$.

Note that any non-zero closed two-sided ideal of the tensor product $A \otimes C(\mathbf{T})$ contains a non-zero element $a \otimes f$. If $\gamma$ is not a norm, there is a non-zero element $a \otimes f$ such that

$$
\gamma\left(a^{*} a \otimes f^{*} f\right)=0
$$

Since $f^{*} f$ dominates $c \chi_{I}$ for some $c>0$ and $I \neq \emptyset$, this contradicts that $\delta$ is a norm. Since $A \odot C(\mathbf{T})$ has a unique $C^{*}$-norm, we obtain that

$$
\gamma(x)=\|x\|, x \in A \odot C(\mathbf{T})
$$

which suffices to conclude the proof.

Proof of Theorem 2.1

For each $p \in \mathbf{R}, t \mapsto e^{i p t}$ can be regarded as an $\alpha$-cocycle in $A_{\infty, \alpha}$. Thus (1) is a special case of (2). We shall prove that (1) implies (2).

Let $u$ be an $\alpha$-cocycle in $A_{\infty, \alpha}$ and $\epsilon>0$. First we choose an $N \in \mathbf{N}$ so that $l(N) / N<$ $\epsilon$. Let $\left\{u_{n}(\cdot)\right\}$ be a sequence of continuous families of unitaries which represents $u$ as in Lemma 2.2. Then it follows that

$$
u_{n}(s) \alpha_{s}\left(u_{n}(t)\right)-u_{n}(s+t)
$$

converges to zero uniformly in $s, t$ on each compact subset of $\mathbf{R}$. Here we may suppose that $u_{n}(0)=1$ and $l\left(u_{n}(N)\right)<\epsilon N$. We choose a sufficiently large $n \in \mathbf{N}$ so that for $s, t \in[0,2 N]$.

$$
\left\|u_{n}(s) \alpha_{s}\left(u_{n}(t)\right)-u_{n}(s+t)\right\|<\epsilon,
$$


and we let $U=u_{n}$. By Lemma 2.2 we may further assume that there is a continuous family $x(t), t \in[0, N]$ of unitaries such that $x(0)=1, x(N)=U(N)$ and for distinct $s, t \in[0, N]$

$$
\|x(s)-x(t)\|<\epsilon|s-t| .
$$

Define a unitary $W$ in $A \otimes C(\mathbf{T})$ by

$$
W(s)=U(N s) \alpha_{N(s-1)}\left(x(N s)^{*}\right)
$$

for $s \in[0,1]$. Since $W(0)=1=W(1), W$ is in fact in $A \otimes C(\mathbf{T})$.

Define a one-parameter automorphism group $\gamma$ on $C(\mathbf{T})$ by $\left(\gamma_{t} f\right)(s)=f(s-t)$. Suppose that $0 \leq s<1$ and $0<t \leq N$. If $N s>t$, then

$$
\left(W \alpha_{t} \otimes \gamma_{t / N}\left(W^{*}\right)\right)(s)=U(N s) \alpha_{N(s-1)}\left(x(N s)^{*} x(N s-t)\right) \alpha_{t}(U(N s-t))^{*} .
$$

Since $\left\|x(N s)^{*} x(N s-t)-1\right\|<\epsilon t$ and $\left\|u(N s)-U(t) \alpha_{t}(U(N s-t))\right\|<\epsilon$, it follows that

$$
\left\|\left(W \alpha_{t} \otimes \gamma_{t / N}\left(W^{*}\right)\right)(s)-U(t)\right\|<\epsilon(t+1) .
$$

If $N s<t$, then

$\left(W \alpha_{t} \otimes \gamma_{t / N}\left(W^{*}\right)\right)(s)=U(N s) \alpha_{N(s-1)}\left(x(N s)^{*}\right) \alpha_{N s}(x(N+N s-t)) \alpha_{t}\left(U(N+N s-t)^{*}\right)$.

Since $\|x(N s)-1\|<\epsilon N s$ and $\|x(N+N s-t)-U(N)\|<\epsilon(t-N s)$, it follows that

$$
\begin{aligned}
\left\|W \alpha_{t} \otimes \gamma_{t / N}\left(W^{*}\right)(s)-U(t)\right\| & <\epsilon t+\left\|U(N s) \alpha_{N s}(U(N)) \alpha_{t}(U(N+N s-t))^{*}-U(t)\right\| \\
& <\epsilon(t+2) .
\end{aligned}
$$

Hence we obtain for $t \in[0, N]$

$$
\left\|W \alpha_{t} \otimes \gamma_{t / N}\left(W^{*}\right)-U(t) \otimes 1\right\|<\epsilon(t+2) .
$$

By (1) there is a unitary $v \in A_{\infty, \alpha}$ such that $\alpha_{t}(v)=e^{-2 \pi i t / N} v$. Let $\left\{v_{n}\right\}$ be a sequence of unitaries in $A$ representing $v$ such that $\left\{v_{n}\right\}$ is equicontinuous with respect to $\alpha$. We define a linear map $\phi_{n}$ of $A \odot C(\mathbf{T})$ into $A$ by

$$
\phi_{n}(a \otimes f)=a f\left(v_{n}\right)
$$

as in Lemma 2.3 .

Note that for $a \in A$ and $f \in C(\mathbf{T})$

$$
\phi_{n} \circ \alpha_{t} \otimes \gamma_{t / N}(a \otimes f)=\alpha_{t}(a) f\left(e^{-2 \pi i t / N} v_{n}\right) \approx \alpha_{t}\left(a f\left(v_{n}\right)\right)
$$

i.e., for $x \in A \odot C(\mathbf{T})$,

$$
\lim \left\|\phi_{n} \circ \alpha_{t} \otimes \gamma_{t / N}(x)-\alpha_{t} \circ \phi_{n}(x)\right\|=0 .
$$


We find a $W_{1} \in A \odot C(\mathbf{T}) \subset A \otimes C(\mathbf{T})$ such that $\left\|W_{1}-W\right\|<\epsilon$ and find an $n \in \mathbf{N}$ such that for $t \in[0, N)$,

$$
\begin{aligned}
& \left\|\phi_{n}\left(W_{1}\right) \phi_{n}\left(W_{1}^{*}\right)-1\right\|<\epsilon+\left\|W_{1} W_{1}^{*}-1\right\|, \\
& \left\|\phi_{n}\left(W_{1}^{*}\right) \phi_{n}\left(W_{1}\right)-1\right\|<\epsilon+\left\|W_{1}^{*} W_{1}-1\right\|, \\
& \left\|\phi_{n} \circ \alpha_{t} \otimes \gamma_{t / N}\left(W_{1}\right)-\alpha_{t} \circ \phi_{n}\left(W_{1}\right)\right\|<\epsilon \\
& \| \phi_{n}\left(W_{1}\right) \phi_{n} \circ \alpha_{t} \otimes \gamma_{t / N}\left(W_{1}^{*}\right)-\phi_{n}\left(W_{1} \alpha \otimes \gamma_{t / N}\left(W_{1}^{*}\right) \|<\epsilon\right. \\
& \left\|\phi_{n}\left(W_{1} \alpha_{t} \otimes \gamma_{t / N}\left(W_{1}^{*}\right)\right)-\phi_{n}(U(t) \otimes 1)\right\|<\epsilon+\left\|W_{1} \alpha_{t} \otimes \gamma_{t / N}\left(W_{1}^{*}\right)-U(t) \otimes 1\right\| .
\end{aligned}
$$

Then for $\tilde{W}=\phi_{n}\left(W_{1}\right)$ we obtain that

$$
\begin{aligned}
\left\|\tilde{W} \alpha_{t}\left(\tilde{W}^{*}\right)-U(t)\right\| & <\epsilon+\left\|\phi_{n}\left(W_{1}\right) \phi_{n} \circ \alpha_{t} \otimes \gamma_{t / N}\left(W_{1}\right)^{*}-U(t)\right\| \\
& <2 \epsilon+\left\|\phi_{n}\left(W_{1} \alpha_{t} \otimes \gamma_{t / N}\left(W_{1}^{*}\right)\right)-\phi_{n}(U(t) \otimes 1)\right\| \\
& <3 \epsilon+\left\|W_{1} \alpha_{t} \otimes \gamma_{t / N}\left(W_{1}^{*}\right)-U(t) \otimes 1\right\| \\
& <5 \epsilon+\left\|W \alpha_{t} \otimes \gamma_{t / N}\left(W^{*}\right)-U(t) \otimes 1\right\| \\
& <7 \epsilon+\epsilon t .
\end{aligned}
$$

Since $\tilde{W}$ is close to a unitary, the unitary $w$ obtained by the polar decomposition of $\tilde{W}$ has the desired properties.

For each sufficiently large $n$ we specify $N, x(t)$ and $v_{m}$ and then construct $w_{n}=w$ in the above way. If $A$ is separable, it is easy to make $\left\{w_{n}\right\}$ central. (We have assumed the separability only for this reason.) This concludes the proof of $(1) \Rightarrow(2)$.

The last statement follows from the same proof as above. We do not need the separability for this statement.

Proposition 2.4 Let $A$ be a unital simple $C^{*}$-algebra and let $\alpha$ be a strongly continuous one-parameter automorphism group with the Rohlin property. Then the crossed product $A \times \mathbf{R}$ is simple.

Proof. The dual action $\hat{\alpha}$ of $\mathbf{R}$ on $A \times \mathbf{R}$ is defined by $\hat{\alpha}_{p}(a)=a, \hat{\alpha}_{p}\left(\lambda_{t}\right)=e^{i p t} \lambda_{t}$ where $a \in A$ and the canonical unitary group $\lambda$ implementing $\alpha$ on $A$ are elements in the multiplier algebra. Then it soon follows that each $\hat{\alpha}_{p}$ is approximately inner, i.e., $\hat{\alpha}_{p}=\lim \operatorname{Ad} u_{n} \mid A \times \mathbf{R}$ where $\left\{u_{n}\right\}$ is a central sequence of unitaries in $A$ with $\lim \| \alpha_{t}\left(u_{n}\right)-$ $e^{-i p t} u_{n} \|=0$ uniformly in $t$ on every compact subset of $\mathbf{R}$. Hence any closed two-sided ideal of $A \times \mathbf{R}$ is left invariant under $\hat{\alpha}$. Since $A$ is simple, this implies that $A \times \mathbf{R}$ is simple.

Proposition 2.5 Let $A$ be a simple non-commutative $n$-torus, i.e., the universal $C^{*}$ algebra generated by $n$ unitaries $u_{1}, \ldots, u_{n}$ with $u_{i} u_{j} u_{i}^{*} u_{j}^{*}=e^{2 \pi i \theta_{i j}} 1 \in \mathbf{C} 1$ such that the anti-symmetric matrix $\Theta=\left(\theta_{i j}\right)$ satisfies that $\Theta m \notin \mathbf{Z}^{n}$ for any non-zero $m \in \mathbf{Z}^{n}$. Let $\alpha$ be a one-parameter automorphism group of $A$ such that

$$
\alpha_{t}\left(u_{j}\right)=e^{2 \pi i p_{j} t} u_{j}
$$


and any $\alpha_{t}$ with $t \neq 0$ is not inner, i.e., $\left(\mathbf{Z}^{n}+\Theta \mathbf{Z}^{n}\right) \cap \mathbf{R} p=\{0\}$ with $p=\left(p_{1}, \cdots, p_{n}\right)^{t}$. Then $\alpha$ has the Rohlin property.

Proof. This is noted in [18] in a different context. Let $q \in \mathbf{R}$. We shall find a sequence $\left\{m_{k}\right\}$ in $\mathbf{Z}^{n}$ such that

$$
\operatorname{dist}\left(\Theta m_{k}, \mathbf{Z}^{n}\right) \rightarrow 0, p^{t} m_{k} \rightarrow q
$$

Then the sequence of unitaries

$$
u^{m_{k}}=u_{1}^{m_{k 1}} u_{2}^{m_{k 2}} \cdots u_{n}^{m_{k n}}
$$

is central and satisfies that $\alpha_{t}\left(u^{m_{k}}\right)-e^{2 \pi i q t} u^{m_{k}} \rightarrow 0$.

This follows since

$$
G=\left\{\left(\Theta m+k, p^{t} m\right) \mid k, m \in \mathbf{Z}^{n}\right\}
$$

is dense in $\mathbf{R}^{n+1}$. To prove the density of $G$ suppose that $\bar{G} \neq \mathbf{R}^{n+1}$. Then since $G$ is a subgroup of $\mathbf{R}^{n+1}$, there must be a non-zero $\xi=\left(\xi_{0}, \xi_{1}\right) \in \mathbf{R}^{n} \times \mathbf{R}$ such that $\langle\xi, g\rangle \in \mathbf{Z}$ for any $g \in \bar{G}$, i.e.,

$$
\left\langle-\Theta \xi_{0}, m\right\rangle+\left\langle\xi_{0}, k\right\rangle+\left\langle\xi_{1} p, m\right\rangle \in \mathbf{Z}
$$

which implies that $\xi_{0} \in \mathbf{Z}^{n}$ and

$$
\left\langle-\Theta \xi_{0}+\xi_{1} p, m\right\rangle \in \mathbf{Z}
$$

Then it follows that $\xi_{1} \neq 0$ and $\xi_{1} p \in \mathbf{Z}^{n}+\Theta \mathbf{Z}^{n}$, a contradiction.

\section{Single automorphisms}

Let $A$ be a unital $C^{*}$-algebra and let $\alpha$ be an automorphism of $A$. Let

$$
A_{\alpha}=\{f \in C[0,1] \otimes A \mid f(1)=\alpha(f(0))\} .
$$

Condition 3.1 There is an increasing function $f: \mathbf{R}_{+} \rightarrow \mathbf{R}_{+}$such that for any $u$ in the connected component of 1 of the unitary group of $A_{\alpha}$ there is a continuous path $\tilde{u}_{s}, s \in[0,1]$ of unitaries in $A_{\alpha}$ such that $\tilde{u}_{0}=u, \tilde{u}_{1}=1$, and the length $l(\tilde{u})$ of $\tilde{u}$ is bounded by $f(L(u))$ where $L(u)$ is the length of $u(s), s \in[0,1]$.

We shall consider this condition later in Propositions 3.4 and 3.5.

Theorem 3.2 Let $A$ be a unital simple $C^{*}$-algebra and let $\alpha$ be an automorphism of $A$. Suppose that Condition 3.1 is satisfied and that for any $\mu \in \mathbf{T}$ there is a unitary $v \in A_{\infty}$ such that $\alpha(v)=\mu v$. Then for any $u$ in the connected component of 1 of the unitary group of $A$ there is a sequence $\left\{v_{n}\right\}$ of unitaries in $A$ such that $u=\lim v_{n} \alpha\left(v_{n}^{*}\right)$. 
Proof. Let $u \in A$ be a unitary in the connected component of 1 and let $u(1)=u$ and $u(k)=u \alpha(u(k-1))$ for $k=2,3, \ldots$, i.e., $u$ is an $\alpha$-cocycle in the sense that $u(k) \alpha^{k}(u(m))=u(k+m)$ for $k, m \in \mathbf{Z}$. Let $v_{0}$ be a rectifiable path of unitaries in $A$ such that

$$
v_{0}(0)=1, v_{0}(1)=u \text {. }
$$

Let, for $k=1,2, \ldots, n-1$,

$$
v_{k}=u(k) \alpha^{k}\left(v_{0}\right) \in C[0,1] \otimes A .
$$

Note that $s \in[0,1] \mapsto v_{0}(s \cdot)^{*} v_{0}(s)(n) \alpha^{n}\left(v_{0}(s \cdot)\right)$ is a path from 1 to $v_{0}^{*} u(n) \alpha^{n}\left(v_{0}\right)$ in the unitary group of $A_{\alpha}$, where $v_{0}(s)(n)$ is defined in the same way as $u(n)$, based on $v_{0}(s)$ instead of $u$, and that the length $L\left(v_{0}^{*} u(n) \alpha^{n}\left(v_{0}\right)\right)$ as a function on $[0,1]$ is at most $2 l\left(v_{0}\right)$, independent of $n$. Using Condition 3.1 let $w_{0}=1, w_{1}, \ldots, w_{n-2}, w_{n-1}=\alpha^{-n}\left(v_{0}^{*} u(n) \alpha^{n}\left(v_{0}\right)\right)$ be a sequence of unitaries in $A_{\alpha}$ such that

$$
\left\|w_{k}-w_{k-1}\right\|<C / n, k=1,2, \ldots, n-1,
$$

where $C=f\left(2 l\left(v_{0}\right)\right)$. For $k=0,1, \ldots, n-2$ let $\tilde{w}_{k} \in C[0,1] \otimes A$ be a unitary such that

$$
\begin{aligned}
& \tilde{w}_{k}(t)=w_{k}(t), t \in[0,1 / 2], \\
& \tilde{w}_{k}(1)=w_{k+1}(1), \\
& \left\|\tilde{w}_{k}-w_{k}\right\|<C / n
\end{aligned}
$$

and let $\tilde{w}_{n-1}=w_{n-1}$.

Let $\tilde{v}_{k}=v_{k} \alpha^{k}\left(\tilde{w}_{k}^{*}\right)$. We define a unitary $v \in C(\mathbf{T}) \otimes A$ as follows: for $t \in[k / n,(k+$ 1) $/ n$ ),

$$
v(t)=\tilde{v}_{k}(n t-k)
$$

Then $v$ is indeed continuous in $t \in \mathbf{T}$ because for $k=0,1, \ldots, n-1$,

$$
\begin{aligned}
& \tilde{v}_{k}(1)=u(k) \alpha^{k}(u) \alpha^{k}\left(w_{k+1}(1)^{*}\right)=u(k+1) \alpha^{k}\left(w_{k+1}(1)^{*}\right) \\
& \tilde{v}_{k+1}(0)=u(k+1) \alpha^{k+1}\left(w_{k+1}(0)^{*}\right)=u(k+1) \alpha^{k}\left(w_{k+1}(1)^{*}\right)
\end{aligned}
$$

and

$$
\begin{aligned}
& \tilde{v}_{0}(0)=1 \\
& \tilde{v}_{n-1}(1)=u(n-1) \alpha^{n-1}(u) \alpha^{-1}\left(u^{*} u(n) \alpha^{n}(u)\right)=u(n) \alpha^{-1}\left(u^{*} u(n+1)\right)=1 .
\end{aligned}
$$

Note also that

$$
\begin{aligned}
\tilde{v}_{k+1} \alpha\left(\tilde{v}_{k}^{*}\right) & =v_{k+1} \alpha^{k+1}\left(\tilde{w}_{k+1}^{*}\right) \alpha\left(v_{k} \alpha^{k}\left(\tilde{w}_{k}\right)^{*}\right)^{*} \\
& =u(k+1) \alpha^{k+1}\left(v_{0}\right) \alpha^{k+1}\left(\tilde{w}_{k+1}^{*} \tilde{w}_{k}\right) \alpha^{k+1}\left(v_{0}^{*}\right) \alpha\left(u(k)^{*}\right) \\
\tilde{v}_{0} \alpha\left(\tilde{v}_{n-1}^{*}\right) & =v_{0} \tilde{w}_{0}^{*} \alpha\left(v_{n-1} \alpha^{n-1}\left(\tilde{w}_{n-1}^{*}\right)\right)^{*} \\
& =v_{0} \tilde{w}_{0}^{*} \alpha^{n}\left(\tilde{w}_{n-1}\right) \alpha^{n}\left(v_{0}^{*}\right) \alpha\left(u(n-1)^{*}\right) \\
& =v_{0} \tilde{w}_{0}^{*} v_{0}^{*} u
\end{aligned}
$$


Thus it follows that

$$
\|v(t) \alpha(v(t-1 / n))-u\|<3 C / n .
$$

Let $\left\{u_{m}\right\}$ be a central sequence of unitaries in $A$ such that $\left\|\alpha\left(u_{m}\right)-e^{-2 \pi i / n} u_{m}\right\| \rightarrow 0$. Define a linear map $\phi_{m}$ of $A \odot C(\mathbf{T})$ into $A$ by

$$
\phi_{m}(a \otimes f)=a f\left(u_{m}\right)
$$

Since $\left\|v \alpha \otimes \gamma_{1 / n}\left(v^{*}\right)-u \otimes 1\right\|<3 C / n$ and $\phi_{m} \circ \alpha \otimes \gamma_{1 / n} \approx \alpha \circ \phi_{m}$, we have, as in the proof of Theorem 2.1, that for a sufficiently large $m$, a unitary $w$ which is close to " $\phi_{m}(v)^{\prime \prime}$ satisfies that

$$
\left\|w \alpha\left(w^{*}\right)-u\right\|<3 C / n
$$

This concludes the proof

We have not proved an obvious adaptation of Theorem 2.1 to this case, i.e., the equivalence of the following two conditions:

1. For any $\mu \in \mathbf{T}$ there is a unitary $v \in A_{\infty}$ such that $\alpha(v)=\mu v$.

2. For any $u$ in the connected component of the unitary group of $A_{\infty}$ there is a unitary $v \in A_{\infty}$ such that $u=v \alpha\left(v^{*}\right)$.

To prove this we would need an obvious condition involving central sequences which is stronger than Condition 3.1, and which we could not prove unless $A$ is an AF algebra. We shall now consider Condition 3.1.

Lemma 3.3 Let $A$ be a unital $C^{*}$-algebra of real rank zero and let $u(s), s \in[0,1]$ be a continuous path of unitaries in $A$ with $[u(0)]=0$ in $K_{1}(A)$. Then for any $\epsilon>0$ there is a continuous function $h$ of $[0,1]$ into the self-adjoint part of $A$ such that for all $s \in[0,1]$

$$
\left\|u(s)-e^{i h(s)}\right\|<\epsilon .
$$

Furthermore if $u(0)=1=u(1)$, then $h$ can be chosen such that $h(0)=0, e^{i h(1)}=1$, and $\|h(t)\|<C_{1} l(u)+C_{2}$ where $C_{1}$ and $C_{2}$ are constants independent of $u$ (depending on $\epsilon$ ).

Proof. For any $\epsilon>0$ there exist a $\delta \in(0, \epsilon / 2)$ and $N \in \mathbf{N}$ such that if $\left\|u_{0}-u_{1}\right\|<\delta$ with $u_{0}, u_{1}$ unitaries of finite spectra, the unitaries

$$
\tilde{u}_{i}=\sum_{k=0}^{2 N-1} e^{\pi i k / N} P_{i}([k / 2 N-1 / 4 N, k / 2 N+1 / 4 N))
$$

where $P_{i}(\cdot)$ is the spectral measure of $u_{i}$ on $\mathbf{T}$, can be connected by a continuous path $\tilde{u}_{t}$ of unitaries such that $\left\|\tilde{u}_{t}-u_{i}\right\|<\epsilon / 2, \operatorname{Sp}\left(\tilde{u}_{t}\right)$ is finite, and $G=\left\{(t, \lambda) \mid \lambda \in \operatorname{Sp}\left(\tilde{u}_{t}\right)\right\}$ looks like: 


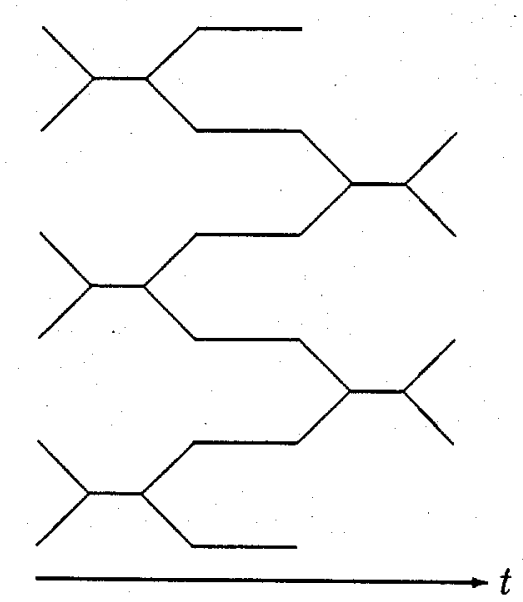

Some of the end points may not exist (all $e^{\pi i k / N}$ 's may not be eigenvalues of $\operatorname{Sp}\left(\tilde{u}_{i}\right)$ ); so some line segments should be removed from the above picture (cf. [2]; here we have used freedom to change the pattern of eigenvalues slightly). In particular, $\left(0, e^{\pi i k / N}\right)$ may be connected to at most four points of $\left(1, e^{\pi i k / N+\pi i l / N}\right), l=-2,-1,0,1,2$ in $G$ as $t$ increases.

Let $L$ be the length $l(u)$ of $u$. If $M \in \mathbf{N}$ satisfies that $L / M<\delta \leq L /(M-1)$ one finds a sequence $t_{0}=0<t_{1}<t_{2}<\cdots<t_{M}=1$ such that for $s, t \in\left[t_{i-1}, t_{i}\right]$

$$
\|u(s)-u(t)\|<\delta \text {. }
$$

Then composing the $\tilde{u}$ constructed for the pair $u\left(t_{i-1}\right), u\left(t_{i}\right)$, we obtain a path $\tilde{u}(s), s \in$ $[0,1]$ such that $\|u(s)-\tilde{u}(s)\|<\epsilon$ and $G_{i}=\left\{(t, \lambda) \mid t \in\left[t_{i-1}, t_{i}\right], \lambda \in \operatorname{Sp}(\tilde{u}(t))\right\}$ satisfies the condition as above. Then starting from $h(0)=-i \log \tilde{u}(0)$ with $\|h(0)\| \leq \pi$ we can continuously define $h(t)=-i \log \tilde{u}(t)$; the definition of $h(t)$ (or the eigenvalues of $h(t))$ is by no means unique and in general the spectral projections of $h(t)$ are finer than those of $\tilde{u}(t)$.

To be more precise, if $\tilde{u}(t)=\sum_{i} \mu_{i}(t) p_{i}(t), p_{i}(t)$ 's are projections with $\sum_{i} p_{i}(t)=1$, and $\mu_{i}(t)$ 's are mutually distinct at a neighbourhood of $t=t_{0}, h(t)$ may be defined as

$$
\sum_{i} \sum_{k}\left(-i \log \mu_{i}(t)+2 \pi k\right) p_{i k}(t)
$$

in that neighbourhood, where $\sum_{k}$ is the sum over a finite set of integers, $\log \mu_{i}(t)$ is defined as a continuous function, and $p_{i k}(t)$ 's are projections with $\sum_{k} p_{i k}(t)=p_{i}(t)$.

If $\mu_{i}(t)$ 's are mutually distinct for $t \leq t_{0}$ except for $\mu_{2 i-1}\left(t_{0}\right)=\mu_{2 i}\left(t_{0}\right)$, and $\tilde{u}(t)=$ $\sum_{i} \mu_{i}^{\prime}(t) p_{i}^{\prime}(t)$ for $t \geq t_{0}$ where $\mu_{i}^{\prime}\left(t_{0}\right)=\mu_{2 i-1}\left(t_{0}\right)=\mu_{2 i}\left(t_{0}\right)$ and $p_{i}^{\prime}\left(t_{0}\right)=p_{2 i-1}\left(t_{0}\right)+p_{2 i}\left(t_{0}\right)$, then $h(t)$ is defined up to $t=t_{0}$ as in $(*)$ and by assuming $\log \mu_{2 i-1}\left(t_{0}\right)=\log \mu_{2 i}(t), h(t)$ may be defined as

$$
\sum_{i} \sum_{k}\left(-i \log \mu_{i}^{\prime}(t)+2 \pi k\right) p_{i k}^{\prime}(t)
$$

where $p_{i k}^{\prime}(t)$ 's are projections with $\sum_{k} p_{i k}^{\prime}(t)=p_{i}^{\prime}(t)$.

If $\tilde{u}(t)=\sum_{i} \mu_{i}^{\prime \prime}(t) p_{i}^{\prime \prime}(t)$ for $t \geq t_{0}, p_{i}^{\prime \prime}(t)$ 's are projections with $\sum_{i} p_{i}^{\prime \prime}(t)=1, \mu_{i}^{\prime \prime}(t)$ 's are mutually distinct for $t \geq t_{0}$ except for $\mu_{2 i+1}^{\prime \prime}\left(t_{0}\right)=\mu_{2 i}^{\prime \prime}\left(t_{0}\right)=\mu_{i}\left(t_{0}\right)$, then $h(t)$ is defined 
up to $t=t_{0}$ and $\sum_{k} p_{i k}\left(t_{0}\right)=p_{2 i+1}^{\prime \prime}\left(t_{0}\right)+p_{2 i}^{\prime \prime}\left(t_{0}\right)$. Let $t_{1}<t_{0}$ be close to $t_{0}$. By the Riesz decomposition property, we find subprojections $q_{i k}$ of $p_{i}^{\prime \prime}\left(t_{0}\right)$ such that

$$
\begin{aligned}
& {\left[p_{i k}\left(t_{1}\right)\right]=\left[q_{2 i+1, k}\right]+\left[q_{2 i, k}\right],} \\
& \sum_{k} q_{i k}=p_{i}^{\prime \prime}\left(t_{0}\right) .
\end{aligned}
$$

Then we find a path $\tilde{p}_{i k}(t), t \in\left[t_{1}, t_{0}\right]$ of projections from $p_{i k}\left(t_{1}\right)$ to $q_{2 i+1, k}+q_{2 i, k}$ at $t=t_{0}$ for each $k$ such that $\sum_{k} \tilde{p}_{i k}(t)=p_{i}(t)$. By using these paths we change $h(t)$ for $t \in\left[t_{1}, t_{0}\right]$ keeping the relation $\tilde{u}(t)=e^{i h(t)}$. Then we can continue to define $h(t)$ for $t>t_{0}$ by finding paths $p_{i k}^{\prime \prime}(t)$ such that $p_{i k}^{\prime \prime}\left(t_{0}\right)=q_{i k}$ and $\sum_{k} p_{i k}^{\prime \prime}(t)=p_{i}^{\prime \prime}(t)$.

If $u(0)=1=u(1)$, we assume that $\tilde{u}(0)=1=\tilde{u}(1)$. Starting with $h(0)=0$, we can estimate the norm of $h(t)$ : If $t_{k-1} \leq t<t_{k}$,

$$
\|h(t)\| \leq 2 \pi k / N
$$

and hence for any $t \in[0,1]$

$$
\|h(t)\| \leq 2 \pi M / N \leq 2 \pi(L / \delta+1) / N=\frac{2 \pi}{\delta N} L+\frac{2 \pi}{N} .
$$

We note that if $K_{0}(A)$ is totally ordered, then the above $h$ can be defined in a unique way by requiring that the ramification can occur at most on one eigenvalue of $\tilde{u}(t)$. (This requirement makes the choice of subprojections $q_{i k}$ unique when we use the Riesz decomposition property in the above proof.) In this case if $u(0)=1=u(1)$ we have that $h(1) \in 2 \pi \mathbf{Z} 1$.

We also note that if $A$ is purely infinite and simple, we can also impose the above requirement, though in this case this does not remove the freedom we have when applying the Riesz decomposition property. (If $\operatorname{Sp}(\tilde{u})$ is full, we can use this freedom to control the norm of $h(t)$ to just over $\pi$.)

Proposition 3.4 Let $A$ be a unital simple $C^{*}$-algebra of real rank zero such that $A$ has a weakly unperforated ordered group with the Riesz decomposition property as $K_{0}(A)$.. and has the cancellation property. Let $\alpha$ be an automorphism of $A$ such that $\alpha_{*}=$ id on $K_{0}(A)$. Then $A_{\alpha}$ satisfies Condition 3.1.

Proof. Let $u \in A_{\alpha}$ be a unitary with $[u]=0$. Since $u(0)$ can be approximated by a unitary of finite spectrum, we may assume that $\operatorname{Sp}(u(0))$ is finite. Let $h=h^{*} \in A$ be such that $\|h\| \leq \pi$ and $u(0)=e^{i h}$. Let

$$
u_{s}(t)=u(t) e^{-i(1-t) s h-i t s \alpha(h)} .
$$

Then $u_{0}=u, u_{1}(0)=1=u_{1}(1)$, the length of $u_{s}, s \in[0,1]$ is $\|h\|$, and the length $L\left(u_{1}\right)$ of $u_{1}$ as a function on $[0,1]$ is at most $2\|h\|$ greater than $L(u)$. Hence we may assume that $u(0)=1=u(1)$ and we can regard $u$ as an element of $(S A)^{+}=(C(0,1) \otimes A)^{+}$. 
Suppose that $u_{s}, s \in[0,1]$ is a path from $u$ to 1 in the unitary group of $A_{\alpha}$. By Lemma $3.3 u_{s}(1)$ can be approximated by $e^{i h(s)}$ with $h(0)=0$ and $e^{i h(1)}=1$. Hence there is another $h^{\prime}(s)$ with $\left\|h^{\prime}(s)\right\| \approx 0$ such that

$$
u_{s}(0)=e^{i h(s)} e^{i h^{\prime}(s)}, h^{\prime}(0)=0=h^{\prime}(1) .
$$

By replacing $u_{s}$ by

$$
t \mapsto u_{s}(t) e^{-i(1-t) h^{\prime}(s)-i t \alpha\left(h^{\prime}(s)\right)} e^{-i(1-t) h(s)-i t \alpha(h(s))},
$$

we can assume that $u_{s}(0)=1$. Thus $u$ is connected to

$$
u^{\prime}: t \mapsto e^{-i(1-t) h(1)-i t \alpha(h(1))}
$$

in $(S A)^{+}$. Let $h(1)=\sum_{k} 2 \pi k p_{k}$, where $\left\{p_{k}\right\}$ is an orthogonal family of projections in $A$. Then the class of $u^{\prime}$ is equal to

$$
\sum_{k} k\left[p_{k}\right]-\sum_{k} k\left[\alpha\left(p_{k}\right)\right]=0
$$

in $K_{1}(S A)$ which is identified with $K_{0}(A)$, where we have used that $\left[p_{k}\right]=\left[\alpha\left(p_{k}\right)\right]$. Hence $u$ (with $u(0)=1=u(1))$ is connected to 1 in the unitary group of $(S A)^{+}$.

By applying Lemma 3.3 to $u$ with $\epsilon=2$, there are continuous functions $h, h^{\prime}$ of $[0,1]$ into the self-adjoint part of $A$ such that $h(0)=0=h^{\prime}(0), h^{\prime}(1)=0,\|h(t)\| \leq$ $C_{1} L+C_{2},\left\|h^{\prime}(t)\right\|<\pi$, and

$$
u(t)=e^{i h(t)} e^{i h^{\prime}(t)} .
$$

Note that $u$ and the unitary $v$ defined by $v(t)=e^{i h(t)}$ can be connected by a path of unitaries in $(S A)^{+}$of length at most $\pi$ and that $v$ and the unitary $w$ defined by $w(t)=e^{i t h(1)}$ can be connected by the path of unitaries $e^{i(1-s) h(t)+i s t h(1)}$ whose length is at most $2\left(C_{1} L+C_{2}\right)$. We shall now show that $w$ can be connected to 1 by a path of unitaries in $(S A)^{+}$. of length at most $4 \pi\left(C_{1} L+C_{2}\right)$.

Let

$$
h(1)=\sum_{k=-K}^{K} 2 \pi k p_{k}
$$

where $\left\{p_{k}\right\}$ are mutually orthogonal projections in $A$ and $|K| \leq C_{1} L+C_{2}$. Since $[w]=0$ in $K_{1}(S A)$, we must have that

$$
\sum k\left[p_{k}\right]=0 \text {. }
$$

Let $K_{0}$ be the maximum $|k|$ with $p_{k} \neq 0$. If $K_{0}=0$ then $h(1)=0$ and there is nothing to prove. If $K_{0}>0$, suppose that $p_{K_{0}} \neq 0$. Since

$$
K_{0}\left[p_{K_{0}}\right]+\sum_{k=-1}^{-K_{0}} k\left[p_{k}\right] \leq 0
$$


it follows that

$$
K_{0}\left[p_{K_{0}}\right] \leq K_{0} \sum_{k=-1}^{-K_{0}}\left[p_{k}\right]
$$

Suppose that $\left[p_{K_{0}}\right] \leq \sum_{k=-1}^{-K_{0}}\left[p_{k}\right]$ which follows from the strict inequality in the above formula by $K_{0}(A)$ being weakly unperforated; then there are subprojections $q_{k}$ of $p_{k}$ for $k=-1, \ldots,-K_{0}$ such that

$$
\left[p_{K_{0}}\right]=\sum_{k=-1}^{K_{0}}\left[q_{k}\right]
$$

By using the cancellation property we then find a partial isometry $W$ such that

$$
W W^{*}=p_{K_{0}}, W^{*} W=\sum_{k=-1}^{-K_{0}} q_{k}
$$

Let

$$
U_{\theta}=\cos \theta\left(p_{K_{0}}+\sum_{k=-1}^{-K_{0}} q_{k}\right)+\sin \theta\left(W-W^{*}\right)+1-p_{K_{0}}-\sum_{k=-1}^{-K_{0}} q_{k}
$$

Then

$$
U_{\theta} e^{2 \pi i t p_{K_{0}}} U_{\theta}^{*} e^{i t h(1)-2 \pi i t p_{K_{0}}}
$$

connects $w$ to $e^{i t h^{\prime}}$ where

$$
h^{\prime}=2 \pi \sum_{k=0}^{K_{0}-1} k p_{k}+2 \pi \sum_{k=-K_{0}}^{-1} k\left(p_{k}-q_{k}\right)+2 \pi \sum_{-K_{0}}^{-1}(k+1) q_{k}+2 \pi\left(K_{0}-1\right)\left(p_{K_{0}}+p_{K_{0}-1}\right) .
$$

If $\left[p_{K 0}\right] \not \leq \sum_{k=-1}^{-K_{0}}\left[p_{k}\right]$, i.e., $K_{0} \geq 2$ and $K_{0}\left[p_{K 0}\right]=K_{0} \sum_{k=-1}^{-K_{0}}\left[p_{k}\right]$ which implies that $p_{k}=0$ for $k=-1, \ldots,-K_{0}+1$, let $p_{K_{0}}^{\prime}$ be a non-zero subprojection of $p_{K_{0}}$. Then $\left[p_{K_{0}}^{\prime}\right] \leq\left[p_{-K_{0}}\right]$, and we apply the above procedure with $p_{K_{0}}^{\prime}$ in place of $p_{K_{0}}$. Then we again apply the above procedure to the resulting $h^{\prime}$ with $p_{K_{0}}-p_{K_{0}}^{\prime}$ in place of $p_{K_{0}}$, to transform $e^{i t h^{\prime}}$ to $e^{i t h^{\prime \prime}}$ with

$$
h^{\prime \prime}=2 \pi \sum_{k=-K_{0}}^{K_{0}-1} k p_{k}^{\prime \prime}
$$

with no $K_{0}$ 'th term.

If $p_{-K_{0}} \neq 0$, we can use a similar argument to remove the $-K_{0}$ 'th term. By repeating this argument we find a path of unitaries which connects $w$ to 1 . Since each argument requires a path of length $\pi$, the resulting path has length at most

$$
4 K \pi \leq 4 \pi\left(C_{1} L+C_{2}\right) \text {. }
$$

Proposition 3.5 Let $A$ be a unital purely infinite simple $C^{*}$-algebra and let $\alpha$ be an automorphism of $A$ such that $\alpha_{*}=i d$ on $K_{0}(A)$. Then $A_{\alpha}$ satisfies Condition 3.1. 
Proof. A purely infinite simple $C^{*}$-algebra has real rank zero [28] and satisfies that any non-zero projections $p$ and $q$ are equivalent if $[p]=[q]$ in $K_{0}(A)$ [11]. Hence this can be proved in the same way as Proposition 3.4.

Actually this case is simpler. When we apply Lemma 3.4 in the proof of the above proposition, we impose the extra condition indicated just after the lemma, and we obtain the self-adjoint $h$ which may end up with

$$
h(1)=2 \pi k p+2 \pi(k+1) q
$$

where $p+q=1$ and

$$
k[p]+(k+1)[q]=0 .
$$

We have to estimate the length of a path which connects $w: w(t)=e^{i t h(1)}$ to 1 . Suppose $k>0$. Then since $[p]=(k+1)[1]$, we find $k+1$ non-zero subprojections $e_{1}, \ldots, e_{k+1}$ of $p$ such that $e_{1}+\cdots e_{k+1}=p$ and $\left[e_{i}\right]=1$. Then $w$ and the unitary $y$ defined by $y(t)=e^{2 \pi(k+1) t\left(1-e_{1}\right)}$ can be connected by a path of length $k \pi$. Since $\left[1-e_{1}\right]=0, y$ can be connected to 1 by a path of length $(k+2) \pi$. Thus the estimate is $(2 k+2) \pi=2\|h(1)\|$. The other case can be treated in a similar way.

\section{Real rank zero $C^{*}$-algebras}

Theorem 4.1 Let $A$ be a unital separable simple $C^{*}$-algebra of real rank zero and let $\alpha$ be a one-parameter automorphism group of $A$. Suppose that $\alpha$ has the Rohlin property and that $A$ has a weakly unperforated ordered group with the Riesz decomposition property as $K_{0}$ and satisfies the cancellation property, or $A$ is purely infinite. Then for any $\epsilon>0$ there is a decreasing sequence $\left\{e_{n}\right\}$ of projections in $A$ and an $\alpha$-cocycle $u$ in $A$ such that

$$
\begin{aligned}
& \operatorname{Ad} u(t) \circ \alpha_{t}\left(e_{n}\right)=e_{n}, \\
& \|u(t)-1\|<\epsilon, t \in[0,1],
\end{aligned}
$$

and the limit of $e_{n}$ in the second dual $A^{* *}$ is a minimal projection.

Proof. Let $\left\{x_{n}\right\}$ be a dense sequence in the unit ball of the self-adjoint part of $A$. Let $\epsilon>0 \quad e_{0}=1, \alpha^{(0)}=\alpha$, and $u_{0}(t)=1$ for all $t \in \mathbf{R}$. We shall construct a decreasing sequence $\left\{e_{n}\right\}$ of non-zero projections in $A$ and a sequence $\left\{u_{n}\right\}$ with $u_{n}$ an $\alpha^{(n-1)}$-cocycle such that

$$
\begin{aligned}
& u_{n}\left(1-e_{n-1}\right)=1-e_{n-1}, \\
& \left\|u_{n}(t)-1\right\|<2^{-n} \epsilon, t \in[0,1], \\
& \alpha^{(n)}=\operatorname{Ad} u_{n}(t) \circ \alpha_{t}^{(n-1)} \\
& \alpha_{t}^{(n-1)}\left(e_{n-1}\right)=e_{n-1}, \\
& D_{e_{n}}\left(e_{n} x_{m} e_{n}\right)<1 / n, m=1,2, \ldots, n,
\end{aligned}
$$


where if $e$ is a projection in $A$ and $h$ is a self-adjoint element of $e . A \epsilon, D_{e}(h)$ denotes $\max \sigma_{e}(h)-\min \sigma_{e}(h)$ with $\sigma_{e}(h)$ the spectrum of $h$ in $e A e$. If this is done let

$$
u(t)=\lim _{n \rightarrow \infty} u_{n}(t) \cdots u_{1}(t)
$$

which exists for all $t \in \mathbf{R}$, is an $\alpha$-cocycle, and satisfies

$$
\|u(t)-1\|<\epsilon, t \in[0,1]
$$

Note also that $\operatorname{Ad} u(t) \circ \alpha_{t}\left(e_{n}\right)=e_{n}$ for all $n$. Let $\phi$ be a state of $A$ such that $\phi\left(e_{n}\right)=1$ for all $n$. Then by the condition that $D_{e_{n}}\left(e_{n} x e_{n}\right) \rightarrow 0$ as $n \rightarrow 0, \phi$ is uniquely determined. Thus $\phi$ is a pure state, which proves that $\lim e_{n}$ is a minimal projection in $A^{* *}$.

Suppose that we have constructed $e_{k}$ 's and $u_{k}$ 's up to $k=n-1$. Let $\beta=\alpha^{(n-1)}$ and let $t_{0}>0$ be such that for $t \in\left[0, t_{0}\right]$,

$$
\left\|\beta_{t}\left(x_{m}\right)-x_{m}\right\|<1 / 2 n
$$

for $m=1,2, \ldots, n$. Let $N \in \mathbf{N}$ and $\delta>0$. Then by Lemma 4.2 below we find a non-zero projection $p$ in $e_{n-1} A e_{n-1}$ such that $D_{p}\left(p \beta_{-l t_{0}}\left(x_{m}\right) p\right)<\delta$ for $m=1, \ldots, n$ and $l=0,1, \ldots, N$. Noting that all non-zero powers of $\beta_{t_{0}}$ are outer and applying Lemma 4.3, we may further assume, by replacing $p$ by a smaller projection, that if $k, l=0,1, \ldots, N$, and $k \neq l$,

$$
\left\|\beta_{k t_{0}}(p) x_{m} \beta_{l t_{0}}(p)\right\|<\delta
$$

for $m=0, \ldots, n$ where $x_{0}=1$. By Lemma 4.4 below we find an orthogonal family $\left\{q_{0}, q_{1}, \ldots, q_{N}\right\}$ of projections and a unitary $v$ such that

$$
\begin{aligned}
& q_{0}=p, \\
& v\left(1-e_{n-1}\right)=1-e_{n-1}, \\
& \operatorname{Ad} v \circ \beta_{t_{0}}\left(q_{k-1}\right)=q_{k}, k=1, \ldots, N, \\
& \left\|\beta_{k t_{0}}(p)-q_{k}\right\|<\epsilon(\delta, N), k=0, \ldots, N, \\
& \|v-1\|<\epsilon(\delta, N),
\end{aligned}
$$

where $\epsilon(\delta, N) \rightarrow 0$ as $\delta \rightarrow 0$. Let $\left\{q_{i j}\right\}$ be matrix units such that

$$
\begin{aligned}
& q_{i i}=q_{i} \\
& q_{i j}=\operatorname{Ad} v \circ \beta_{t_{0}}\left(q_{i-1, j-1}\right), i, j=1, \ldots, N .
\end{aligned}
$$

Let

$$
E=\frac{1}{N+1} \sum_{i, j=0}^{N} q_{i j} .
$$

Then $E$ is a projection in $e_{n-1} A e_{n-1}$ and satisfies that

$$
\left\|E-\beta_{t_{0}}(E)\right\|<2 / \sqrt{N}+2 \epsilon(\delta, N) \equiv \epsilon_{1}
$$


and that for $\lambda_{k} \in \sigma_{q_{k}}\left(q_{k} x_{m} q_{k}\right)$,

$$
\begin{aligned}
& \left\|E x_{m} E-\left(\frac{1}{N+1} \sum_{l=0}^{N} \lambda_{l}\right) E\right\| \\
& \leq\left\|\frac{1}{(N+1)^{2}} \sum_{l \neq i} q_{k l} x_{m} q_{i j}\right\|+\left\|\frac{1}{(N+1)^{2}} \sum q_{k l} x_{m} q_{l j}-\left(\frac{1}{N+1} \sum_{l=0}^{N} \lambda_{l}\right) E\right\| \\
& <(N+1)^{2} \delta^{\prime}+\left\|\frac{1}{(N+1)^{2}} \sum q_{k l}\left(x_{m}-\lambda_{l}\right) q_{l j}\right\| \\
& \leq(N+1)^{2} \delta^{\prime}+\sum_{l=0}^{N} D_{q_{l}}\left(q_{l} x_{m} q_{l}\right)
\end{aligned}
$$

where $\delta^{\prime}=\delta+2 \epsilon(\delta, N)$ and

$$
\begin{aligned}
D_{q_{l}}\left(q_{l} x_{m} q_{l}\right) & \leq D_{p}\left(p \beta_{-l t_{0}}\left(x_{m}\right) p\right)+3 \epsilon(\delta, N) \\
& \leq \delta+3 \epsilon(\delta, N) .
\end{aligned}
$$

Thus we have that

$$
D_{E}\left(E x_{m} E\right) \leq(N+1)(N+2)(\delta+3 \epsilon(\delta, N)) \equiv \epsilon_{2} .
$$

Both $\epsilon_{1}$ and $\epsilon_{2}$ defined above can be made arbitrarily small by making $N$ sufficiently large and then $\delta>0$ sufficiently small.

By Lemma 4.5 there is a $\beta$-cocycle $u$ such that

$$
\operatorname{Ad} u_{t} \circ \beta_{t}(p)=p
$$

Let $v(0)=1$ and let for $k=1,2, \ldots$

$$
v(k)=v \beta_{t_{0}}(v) \cdots \beta_{t_{0}}^{k-1}(v)
$$

and define

$$
w_{t}=\sum_{j=0}^{N} \beta_{t}(v(j)) \beta_{j t_{0}}\left(u_{t}^{*} p\right) v(j)^{*} .
$$

Since $\beta_{t}(v(j)) \beta_{j t_{0}}\left(u_{t}^{*} p\right) v(j)^{*}$ is a partial isometry with initial projection $q_{j}$ and final projection $\beta_{t}\left(q_{j}\right), w_{t}$ is also a partial isometry. Note that

$$
w_{t} E w_{t}^{*}=\frac{1}{N+1} \sum \beta_{t}(v(i)) \beta_{i t_{0}}\left(u_{t}^{*} p\right) v(i)^{*} q_{i j} v(j) \beta_{j t_{0}}\left(u_{t}^{*} p\right) \beta_{t}\left(v(j)^{*}\right)
$$

We assume that

$$
q_{10}=v u_{t_{0}}^{*} p
$$


Then

$$
\begin{aligned}
q_{i 0} & =\operatorname{Ad} v(i-1) \circ \beta_{(i-1) t_{0}}\left(q_{10}\right) \operatorname{Ad} v(i-2) \circ \beta_{(i-2) t_{0}}\left(q_{10}\right) \cdots q_{10} \\
& =v(i-1) \beta_{(i-1) t_{0}}\left(q_{10}\right) \beta_{(i-2) t_{0}}\left(v^{*} q_{10}\right) \cdots \beta_{t_{0}}\left(v^{*} q_{10}\right) v^{*} q_{10} \\
& =v(i) u_{i t_{0}}^{*} p
\end{aligned}
$$

and so

$$
q_{i j}=v(i) u_{i t_{0}}^{*} p u_{j t_{0}} v(j)^{*}
$$

By using this we obtain that

$$
\begin{aligned}
w_{t} E w_{t}^{*} & =\frac{1}{N+1} \sum \beta_{t}(v(i)) \beta_{i t_{0}}\left(u_{t}^{*} p\right) u_{i t_{0}}^{*} p u_{j t_{0}} \beta_{j t_{0}}\left(u_{t}^{*} p\right)^{*} \beta_{t}\left(v(j)^{*}\right) \\
& =\frac{1}{N+1} \sum \beta_{t}(v(i)) u_{i t_{0}+t}^{*} p u_{j t_{0}+t} \beta_{t}\left(v(j)^{*}\right) \\
& =\frac{1}{N+1} \sum \beta_{t}(v(i)) \beta_{t}\left(u_{i t_{0}}^{*}\right) \beta_{t}(p) \beta_{t}\left(u_{j t_{0}}\right) \beta_{t}\left(v(j)^{*}\right) \\
& =\beta_{t}(E)
\end{aligned}
$$

Note that

$$
\begin{aligned}
w_{t_{0}} E & \doteq \frac{1}{N+1} \sum_{i, j=0}^{N} \beta_{t_{0}}(v(i)) \beta_{i t_{0}}\left(u_{t_{0}}^{*} p\right) u_{i t_{0}}^{*} p u_{j t_{0}} v(j)^{*} \\
& =\frac{1}{N+1} \sum v^{*} v(i+1) u_{(i+1) t_{0}}^{*} p u_{j t_{0}} v(j)^{*}
\end{aligned}
$$

and so

$$
v w_{t_{0}} E-E=\frac{1}{N+1} \sum_{j=0}^{N} v(N+1) u_{(N+1) t_{0}}^{*} p u_{j t_{0}} v(j)^{*}-\frac{1}{N+1} \sum_{j=0}^{N} p u_{j t_{0}} v(j)^{*}
$$

Hence

$$
\left\|v w_{t_{0}} E-E\right\| \leq \frac{2}{\sqrt{N+1}}
$$

and thus $E w_{t_{0}} E$ is invertible and close to $E$. By Lemma 4.6 below there is a $\beta$-cocycle $u$ in $A$ such that

$$
\begin{aligned}
& u(t)\left(1-e_{n-1}\right)=1-e_{n-1} \\
& \operatorname{Ad} u(t) \circ \beta_{t}(E)=E \\
& \left\|u\left(t_{0}\right)-1\right\|<\gamma\left(\epsilon_{1}\right)
\end{aligned}
$$

where $\gamma(\epsilon) \rightarrow 0$ as $\epsilon \rightarrow 0$ as in the lemma.

Let $x(t), t \in\left[0, t_{0}\right]$ be a path of unitaries in $A$ such that

$$
\begin{aligned}
& x(0)=1, x\left(t_{0}\right)=u_{t_{0}} \\
& x(t)\left(1-e_{n-1}\right)=1-e_{n-1} \\
& \|x(s)-x(t)\|<\frac{\gamma\left(\epsilon_{1}\right)}{t_{0}}|s-t|, s, t \in\left[0, t_{0}\right] .
\end{aligned}
$$


Define a unitary $W \in A \otimes C(\mathbf{T})$ by

$$
W(s)=u\left(t_{0} s\right) \beta_{t_{0}(s-1)}\left(x\left(t_{0} s\right)^{*}\right) .
$$

Then

$$
\begin{aligned}
\left(W^{*}(E \otimes 1) W\right)(s) & =W^{*}(s) E W(s) \\
& =\beta_{t_{0}(s-1)}\left(x\left(t_{0} s\right)\right) u\left(t_{0} s\right)^{*} E u\left(t_{0} s\right) \beta_{t_{0}(s-1)}\left(x\left(t_{0} s\right)^{*}\right) \\
& =\beta_{t_{0}(s-1)}\left(x\left(t_{0} s\right)\right) \beta_{t_{0} s}(E) \beta_{t_{0}(s-1)}\left(x\left(t_{0} s\right)^{*}\right)
\end{aligned}
$$

and for $\lambda \in \sigma_{E}\left(E x_{m} E\right)$

$$
\begin{aligned}
& \left\|W^{*}(E \otimes 1) W\left(x_{m} \otimes 1\right) W^{*}(E \otimes 1) W-\lambda W^{*}(E \otimes 1) W\right\| \\
& =\max _{s \in[0,1]}\left\|\beta_{t_{0} s}(E) \beta_{t_{0}(s-1)}\left(x\left(t_{0} s\right)^{*}\right) x_{m} \beta_{t_{0}(s-1)}\left(x\left(t_{0} s\right)\right) \beta_{t_{0} s}(E)-\lambda \beta_{t_{0} s}(E)\right\| \\
& \leq 2 \gamma\left(\epsilon_{1}\right)+1 / 2 n+\epsilon_{2} .
\end{aligned}
$$

As in the proof of Theorem 2.1,

$$
W \beta_{t_{0} t} \otimes \gamma_{t}\left(W^{*}\right)(s) \approx u\left(t_{0} t\right)
$$

with error up to $\gamma\left(\epsilon_{1}\right) t$. This is true for $t \in[0,1]$; then it follows that for all $t \in \mathbf{R} \backslash\{0\}$,

$$
\left\|W \beta_{t} \otimes \gamma_{t / t_{0}}\left(W^{*}\right)-u(t) \otimes 1\right\|<\frac{\gamma\left(\epsilon_{1}\right)}{t_{0}}|t| .
$$

Now we use an approximate homomorphism $\left\{\phi_{m}\right\}$ of $A \odot C(\mathbf{T})$ into $A$ as defined in Lemma 2.3 such that $\phi_{m} \circ \beta_{t_{0} t} \otimes \gamma_{t} \approx \beta_{t_{0} t} \circ \phi_{m}$ and we let $w$ be a unitary obtained from a suitable image of $W$ in $A$ so that the following conditions are satisfied: for $e_{n}=w E w^{*}$,

$$
\begin{aligned}
& \left\|\beta_{t}\left(e_{n}\right)-e_{n}\right\|<\frac{\gamma\left(\epsilon_{1}\right)}{t_{0}}(|t|+1), \\
& D_{e_{n}}\left(e_{n} x_{m} e_{n}\right)<1 / n, m=1, \ldots, n .
\end{aligned}
$$

By Lemma 4.7 we then find a $\beta$-cocycle $u_{n}$ such that

$$
\begin{aligned}
& u_{n}(t)\left(1-e_{n-1}\right)=1-e_{n-1}, \\
& \operatorname{Ad} u_{n}(t) \circ \beta_{t}\left(e_{n}\right)=e_{n}, \\
& u_{n}(t) \approx 1
\end{aligned}
$$

This concludes the proof.

Lemma 4.2 Let $\left\{x_{1}, \ldots, x_{n}\right\}$ be a finite sequence of self-adjoint elements in A. For any $\epsilon>0$ there is a non-zero projection $p$ in $A$ such that $D_{p}\left(p x_{m} p\right)<\epsilon, m=1, \ldots, n$. 
Proof. Here we use the assumption that $A$ has real rank zero (cf. [7]). If $D_{1}\left(x_{1}\right)=$ $\max \sigma_{1}\left(x_{1}\right)-\min \sigma_{1}\left(x_{1}\right) \geq \epsilon$, let $\lambda \in \sigma_{1}\left(x_{1}\right), f$ a non-zero non-negative continuous function on $\mathbf{R}$ such that supp $f \subset(\lambda-\epsilon / 2, \lambda+\epsilon / 2)$, and let $p_{1}$ be a non-zero projection in the hereditary $C^{*}$-subalgebra generated by $f\left(x_{1}\right)$. Then $D_{p_{1}}\left(p_{1} x_{1} p_{1}\right)<\epsilon$. If $\sigma_{p_{1}}\left(p_{1} x_{2} p_{1}\right) \geq \epsilon$, we repeat this procedure for $p_{1} x_{2} p_{1} \in p_{1} A p_{1}$ in place of $x_{1} \in A$ to obtain a non-zero subprojection $p_{2}$ of $p_{1}$ such that $D_{p_{2}}\left(p_{2} x_{2} p_{2}\right)<\epsilon$. Note that $D_{p_{2}}\left(p_{2} x_{1} p_{2}\right) \leq D_{p_{1}}\left(p_{1} x_{1} p_{1}\right)<$ $\epsilon$. We repeat this $n-2$ more times to obtain a projection $p=p_{n}$.

Lemma 4.3 Let $\beta$ be an automorphism of $A$ such that $\beta^{k}$ is outer for any $k=1, \ldots, N$, and let $\left\{x_{1}, \ldots, x_{n}\right\}$ be a finite sequence in $A$. For any non-zero projection $e$ in $A$ and $\epsilon>0$ there exists a non-zero subprojection $p$ of $e$ such that for $k \neq l \in\{0,1, \ldots, N\}$ and $m=1, \ldots, n$,

$$
\left\|\beta^{k}(p) x_{m} \beta^{l}(p)\right\|<\epsilon .
$$

Proof. This follows from [15]. By using the assumption that $A$ has real rank zero, we can take for $p$ a projection instead of a positive element of norm one.

Lemma 4.4 For each $N \in \mathbf{N}$ and sufficiently small $\delta>0$ there exists an $\epsilon(\delta, N)>0$ such that $\lim _{\delta \rightarrow 0} \epsilon(\delta, N)=0$ and the following conditions are satisfied: For any non-zero projection $p$ in $A$ with

$$
\left\|p \beta^{k}(p)\right\|<\delta, k=1,2, \ldots, N
$$

where $\beta$ is an automorphism of $A$, there is an orthogonal family $\left\{q_{0}, q_{1}, \ldots, q_{N}\right\}$ of projections in $A$ and a unitary $v \in A$ such that

$$
\begin{aligned}
& q_{0}=p, \\
& \operatorname{Ad} v \circ \beta\left(q_{k-1}\right)=q_{k}, \\
& \left\|\beta^{k}(p)-q_{k}\right\|<\epsilon(\delta, N), \\
& \|v-1\|<\epsilon(\delta, N)
\end{aligned}
$$

for $k=1, \ldots, N$.

Proof. This is standard. After construction $q_{0}, q_{1}, \ldots, q_{k-1}$, we construct $q_{k}$, by functional calculus, from

$$
\left(1-q_{0}-\cdots-q_{k-1}\right) \beta^{k}(p)\left(1-q_{0}-\cdots-q_{k-1}\right)
$$

which is close to $\beta^{k}(p)$. If $q_{0}, \ldots, q_{N}$ are obtained, the unitary $v$ is obtained, by polar decomposition, from

$$
\sum_{k=0}^{N} q_{k} \beta^{k}(p)+\left(1-\sum_{k=0}^{N} q_{k}\right)\left(1-\sum_{k=0}^{N} \beta^{k}(p)\right) .
$$


Lemma 4.5 For any projection $E$ in $A$ there is an $\alpha$-cocycle $u$ in $A$ such that

$$
\operatorname{Ad} u(t) \circ \alpha_{t}(E)=E \text {. }
$$

Proof. Let $E^{\prime}$ be a projection in $A$ such that $\left\|E-E^{\prime}\right\|<1 / 2$ and $E^{\prime}$ is in the domain of the generator $\delta$ of $\alpha$. Let $W$ be the unitary obtained by the polar decomposition of

$$
E E^{\prime}+(1-E)\left(1-E^{\prime}\right)
$$

Then $W E^{\prime} W=E$. Let $h=\left[\delta\left(E^{\prime}\right), E^{\prime}\right]=\delta\left(E^{\prime}\right) E^{\prime}-E^{\prime} \delta\left(E^{\prime}\right)$. Then $\left[h, E^{\prime}\right]=\delta\left(E^{\prime}\right)$ and so $\left(\delta-\delta_{h}\right)\left(E^{\prime}\right)=0$ where $\delta_{h}(x)=[h, x]$. Define an $\alpha$-cocycle $v$ by

$$
\frac{d}{d t} v(t)=-v(t) \alpha_{t}(h), v(0)=1
$$

Then it follows that $\operatorname{Ad} v(t) \circ \alpha_{t}\left(E^{\prime}\right)=E^{\prime}$. Let $u(t)=W v(t) \alpha_{t}\left(W^{*}\right)$. Then $u$ is the desired $\alpha$-cocycle.

Lemma 4.6 For each small $\epsilon>0$ there exists a $\gamma(\epsilon)>0$ such that $\lim _{\epsilon \rightarrow 0} \gamma(\epsilon)=0$ and the following conditions are satisfied: For each projection $E$ in $A$ and an $\alpha$-cocycle $u$ in A with

$$
\begin{aligned}
& \left\|\alpha_{t_{0}}(E)-E\right\|<\epsilon \text { for some } t_{0}>0 \\
& \operatorname{Ad} u(t) \circ \alpha_{t}(E)=E, \\
& E u\left(t_{0}\right) E \text { is connected to } 1 \text { in the invertible elements of } E A E
\end{aligned}
$$

there is an $\alpha$-cocycle $v$ in $A$ such that

$$
\begin{aligned}
& \operatorname{Ad} v(t) \circ \alpha_{t}(E)=E \\
& \left\|v\left(t_{0}\right)-1\right\|<\gamma(\epsilon)
\end{aligned}
$$

Proof. There exists a unitary $W$ such that $W \approx 1$ and $\operatorname{Ad} W \circ \alpha_{t_{0}}(E)=E$. Since $\left[u\left(t_{0}\right) W^{*} E\right]=0$ in $K_{1}(E A E)$ and $\operatorname{Ad} W \circ \alpha_{t_{0}} \mid E A E$ has the stability by 3.2 , there exists, for any $\epsilon^{\prime}>0$, a unitary $V_{1}$ in $E A E$ such that

$$
\left\|u\left(t_{0}\right) W^{*} E-V_{1}^{*} \operatorname{Ad} W \circ \alpha_{t_{0}}\left(V_{1}\right)\right\|<\epsilon^{\prime} .
$$

In the same way there is a unitary $V_{2}$ in $(1-E) A(1-E)$ such that

$$
\left\|u\left(t_{0}\right) W^{*}(1-E)-V_{2}^{*} \operatorname{Ad} W \circ \alpha_{t_{0}}\left(V_{2}\right)\right\|<\epsilon^{\prime} .
$$

Let

$$
v(t)=\left(V_{1}+V_{2}\right) u(t) \alpha_{t}\left(V_{1}+V_{2}\right)^{*}
$$

Then $v$ is an $\alpha$-cocycle and

$$
\begin{aligned}
& \operatorname{Ad} v(t) \circ \alpha_{t}(E)=E \\
& \left\|v\left(t_{0}\right)-1\right\|<\epsilon^{\prime}+3\|W-1\| .
\end{aligned}
$$


Lemma 4.7 Let $\epsilon>0$ be sufficiently small and e a non-zero projection in $A$ such that $\left\|\alpha_{t}(e)-e\right\|<\epsilon(|t|+1)$. Then there exists an $\alpha$-cocycle $u$ such that

$$
\begin{aligned}
& \operatorname{Ad} u(t) \circ \alpha_{t}(e)=e \\
& \|u(t)-1\|<\delta(\epsilon), t \in[0,1]
\end{aligned}
$$

where $\delta(\epsilon) \rightarrow 0$ as $\epsilon \rightarrow 0$.

Proof. Let $f$ be a non-negative $\mathrm{C}^{\infty}$ function on $\mathbf{R}$ with compact support such that $\int f(t) d t=1$. Let

$$
C_{1}=\int|t| f(t) d t, C_{2}=\int\left|f^{\prime}(t)\right| d t
$$

Then for $f_{\gamma}(t)=f(\gamma t) \gamma$ with $\gamma>0$,

$$
\int f_{\gamma}(t) d t=1, \int|t| f_{\gamma}(t) d t=C_{1} / \gamma, \int\left|f_{\gamma}^{\prime}(t)\right| d t=\gamma C_{2}
$$

Let $h$ be a $C^{\infty}$-function on $\mathbf{R}$ with compact support such that $h(t)=0$ on $(-\infty, 1 / 2-$ $\left.1 / 2^{3 / 2}\right]$ and $h(t)=1$ on $\left[1 / 2+1 / 2^{3 / 2}, 1\right]$. Let $\gamma>0$ be such that

$$
\epsilon\left(C_{1} / \gamma+1\right)=\sqrt{\epsilon} \text { or } \gamma=\frac{C_{1} \sqrt{\epsilon}}{1-\sqrt{\epsilon}} .
$$

Let

$$
x=\int f_{\gamma}(t) \alpha_{t}(e) d t .
$$

Then $\|x-e\|<\epsilon\left(C_{1} / \gamma+1\right)=\sqrt{\epsilon},\|x\| \leq 1$, and so $\left\|x^{2}-x\right\| \leq 2\|x-e\|<2 \sqrt{\epsilon}$. If $2 \sqrt{\epsilon} \leq 1 / 8$, then $\operatorname{Sp}(x) \subset\left[0,1 / 2-1 / 2^{3 / 2}\right] \cup\left[1 / 2+1 / 2^{3 / 2}, 1\right]$ and $p=h(x)$ is a projection such that

$$
\|p-x\| \leq(1-\sqrt{1-8 \sqrt{\epsilon}}) / 2 \leq 4 \sqrt{\epsilon}
$$

Let $\delta$ be the generator of $\alpha$, and let

$$
\hat{h}(p)=\frac{1}{2 \pi} \int h(t) e^{-i p t} d t .
$$

Since

$$
h(x)=\int \hat{h}(p) e^{i p x} d p
$$

it follows that

$$
\|\delta(p)\| \leq C_{3}\|\delta(x)\| \leq C_{2} C_{3} \gamma=\frac{C_{1} C_{2} C_{3} \sqrt{\epsilon}}{1-\sqrt{\epsilon}}
$$

where

$$
C_{3}=\int|\hat{h}(p) p| d p
$$


(See $[6,27]$ for details.) Since $\|p-e\|<5 \sqrt{\epsilon}$, there is a unitary $w$ such that $w p w^{*}=e$ and $\|w-1\|<30 \sqrt{\epsilon}$ (if $10 \sqrt{\epsilon}<1 / 2$ ). By the proof of 4.5 there is an $\alpha$-cocycle $v$ such that

$$
\begin{aligned}
& \|v(t)-1\| \leq 2\|\delta(p)\||| t \mid \\
& \operatorname{Ad} v(t) \circ \alpha_{t}(p)=p
\end{aligned}
$$

Then $u(t)=w v(t) \alpha_{t}\left(w^{*}\right)$ is the desired $\alpha$-cocycle.

We present examples concerning the conclusion of Theorem 4.1; there is a non-unital purely infinite simple $C^{*}$-algebra $B$ and a periodic one-parameter automorphism group $\beta$ of $B$ with period 1 such that if $e$ is a non-zero projection in $B$ then $\|\Delta(e)\| \geq 1$, where $\Delta$ is the generator of $\beta$, and that there is a unital purely infinite simple $C^{*}$-algebra and a one-parameter automorphism group $\alpha$ with $\alpha_{1}$ inner such that the conclusion of Theorem 4.1 does not hold. Note that for this periodic $\beta$ there are many invariant pure states [16].

Let $O_{n}$ be the Cuntz algebra generated by $n$ isometries $S_{1}, \ldots, S_{n}[10]$ and let $\alpha$ be a one-parameter automorphism group of $O_{n}$ such that

$$
\alpha_{t}\left(S_{i}\right)=e^{i p_{i} t} S_{i}
$$

If $\left\{p_{1}, \ldots, p_{n},-p_{i}\right\}$ generates $\mathbf{R}$ as a closed subsemigroup for any $i=1, \ldots, n$, then the crossed product $O_{n} \times_{\alpha} \mathbf{R}$ is simple [14] and furthermore if $p_{i}$ 's are all positive (or all negative), $O_{n} \times{ }_{\alpha} \mathbf{R}$ has no non-zero projections [21]. (Since $O_{n}$ has a KMS state for $\alpha$ in this case, $\alpha$ does not have the Rohlin property. We could not decide whether $\alpha$ has the Rohlin property in the other cases.) Let $A$ be the crossed product of $O_{n}$ by $\alpha_{n}, n \in \mathbf{Z}$. Then $A$ is a unital purely infinite simple $C^{*}$-algebra [22]. We extend the action $\alpha$ to an action $\bar{\alpha}$ on $A$ in a natural way; then $\bar{\alpha}_{1}$ is inner, i.e., $\bar{\alpha}_{1}=\operatorname{Ad} U$ where $U$ is the canonical unitary in $A$ which implements $\alpha_{1}$ on $O_{n}$. Let $f$ be a continuous function on $\mathbf{T}$ with supp $f$ contained in a small neighbourhood of 0 , and let $B$ be the hereditary $C^{*}$-subalgebra of $A$ generated by $f(U)$. Then $\bar{\alpha}_{1} \mid B$ is close to the identity with $\delta=\log \left(\bar{\alpha}_{1} \mid B\right)$ well-defined as $\mathrm{a}^{*}$-derivation ([25], 8.7.7). Let $\Delta$ be the generator of $\bar{\alpha} \mid B$ and let $\beta$ be the one-parameter automorphism group of $B$ generated by $\Delta_{1}=\Delta-\delta$. Then, since $\delta$ commutes with $\alpha$ it follows that $\beta_{1}=i d$. Since $A \times_{\bar{\alpha}} \mathbf{R} \cong O_{n} \times_{\alpha} \mathbf{R} \otimes C(\mathbf{T})$ and $B \times_{\bar{\alpha}} \mathbf{R} \cong B \times_{\beta} \mathbf{R}$ is a continuous field over $\mathbf{T}$ to the $C^{*}$-algebra $B \times_{\beta} \mathbf{T}$, it follows that $B \times_{\beta} \mathbf{T}$ is isomorphic to a hereditary $C^{*}$-subalgebra of $O_{n} \times \mathbf{R}$. Hence it also follows that the fixed point algebra $B^{\beta}$ is isomorphic to a hereditary $C^{*}$-subalgebra of $O_{n} \times \mathbf{R}$. This implies that for any non-zero projection $e$ in the domain of the generator $\Delta_{1}=\Delta-\delta$,

$$
\left\|\Delta_{1}(e)\right\| \geq 1
$$

Because if $\left\|\Delta_{1}(e)\right\|<1$ then let $h=\left[\Delta_{1}(e), e\right]$ and let $\Delta_{2}=\Delta_{1}-\delta_{h}$ with $\delta_{h}(x)=[h, x]$. Then for $t>0$

$$
\left\|e^{t \Delta_{2}}-e^{t \Delta_{1}}\right\|=\left\|\int_{0}^{1} \frac{d}{d s} e^{s t \Delta_{2}} e^{(1-s) t \Delta_{1}} d s\right\| \leq 2\|h\| t .
$$


Since $\|h\|<1$ it followas that $\left\|e^{\Delta_{2}}-i d\right\|<2$. Then $\delta_{1}=\log \left(e^{\Delta_{2}}\right)$ can be defined as a ${ }^{*}$-derivation. Then for $\Delta_{3}=\Delta_{2}-\delta_{1}=\Delta_{1}-\delta_{h}-\delta_{1}$, we obtain that $\Delta_{3}(e)=\epsilon$ and that

$$
e^{\Delta_{3}}=e^{\Delta_{2}} e^{-\delta_{1}}=i d \text {. }
$$

Since $B \times{ }_{\beta_{3}} \mathbf{T} \cong B \times_{\beta} \mathbf{T}$ with $\beta_{3 t}=e^{t \Delta_{3}}, B^{\beta_{3}}$ is isomorphic to a hereditary $C^{*}$-subalgebra of $O_{n} \times \mathbf{R}$. Since $B^{\beta_{3}}$ has the non-zero projection $e$, this is a contradiction.

We assert that the conclusion of Theorem 4.1 does not hold for $(A, \bar{\alpha})$ above. Suppose that there exist a decreasing sequence $\left\{e_{n}\right\}$ of projections and an $\bar{\alpha}$-cocycle $u$ such that

$$
\operatorname{Ad} u(t) \circ \bar{\alpha}_{t}\left(e_{n}\right)=e_{n},
$$

and the limit of $\left\{e_{n}\right\}$ is a minimal projection in $A^{* *}$. Let $\phi$ be a pure state of $A$ such that $\phi\left(e_{n}\right)=1$ for all $n$. There is a one-parameter unitary group $U$ implementing $\bar{\alpha}$ in the GNS representation $\pi_{\phi}$ associated with $\phi$ such that $u(t) U_{t} \xi=\xi$ where $\pi_{\phi}$ is omitted and $\xi$ is the cyclic vector associated with $\phi$. For any $\epsilon>0$ we can find an $\alpha$-cocycle $v$ in $B+\mathbf{C} 1$ and a unit vector $\eta$ in the range of $B$ such that

$$
\begin{aligned}
& \|v(t)-1\|<\epsilon, t \in[0,1], \\
& v(t) U_{t} \eta=e^{i p t} \eta
\end{aligned}
$$

for some $p \in \mathbf{R}$ (cf. [18]). By Kadison's transitivity theorem there is an $x \in A$ such that $x \xi=\eta$ and $\|x\|=1$. Let $f$ be a continuous non-negative function on $\mathbf{R}$ with integral 1 and let

$$
x_{f}=\int e^{-i p t} f(t) v(t) \bar{\alpha}_{t}(x) u(t)^{*} d t
$$

Then $x_{f} \xi=\eta$ and $\left\|x_{f}\right\|=1$. Thus we may suppose that

$$
\left\|e^{i p t} v(t) \bar{\alpha}_{t}(x) u(t)^{*}-x\right\|<\epsilon, t \in[0,1] .
$$

We can now see that $\left\{x e_{n} x^{*}\right\}$ are approximately projections in $B$ (i.e., there is a sequence $\left\{p_{n}\right\}$ of projections in $B$ such that $\left\|x e_{n} x^{*}-p_{n}\right\| \rightarrow 0$ ) (cf. [22]) and that

$$
\left\|\operatorname{Ad} v(t) \circ \bar{\alpha}_{t}\left(x e_{n} x^{*}\right)-x e_{n} x^{*}\right\|<2 \epsilon, t \in[0,1] .
$$

Hence $\left\|\bar{\alpha}_{t}\left(x e_{n} x^{*}\right)-x e_{n} x^{*}\right\|<4 \epsilon, t \in[0,1]$. This implies that $B$ contains an almost $\bar{\alpha}$-invariant projection (cf. 4.6 ), which is a contradiction if $\|\delta\|$ is sufficiently small.

Theorem 4.8 Let $A$ be a unital separable purely infinite simple $C^{*}$-algebra and let $\alpha$ be a one-parameter automorphism group of $A$. If $\alpha$ has the Rohlin property then the crossed product $A \times_{\alpha} \mathbf{R}$ is a purely infinite simple $C^{*}$-algebra.

Proof. The simplicity is shown in Proposition 2.4.

Let $e_{1}, e_{2}$ be non-zero projections in $A$ such that $e_{1} e_{2}=0$ and $\left[e_{i}\right]=[1]$. By 4.5 we obtain an $\alpha$-cocycle $u$ in $A$ such that

$$
\operatorname{Ad} u(t) \circ \alpha_{t}\left(e_{i}\right)=e_{i}
$$


By replacing $\alpha$ by $\operatorname{Ad} u(t) \circ \alpha_{t}$, we assume that $\alpha_{t}\left(e_{i}\right)=e_{i}$. Let $U$ be an isometry in $A$ such that $U U^{*}=e_{1}$. Let $p>0$ and define an $\alpha$-cocycle $u$ by

$$
u(t)=e^{-i p t} U^{*} \alpha_{t}(U)
$$

Then for any $\epsilon>0$ there exists a unitary $V$ in $A$ such that

$$
\left\|u(t)-V \alpha_{t}\left(V^{*}\right)\right\|<\epsilon, t \in[0,1]
$$

Thus for $U_{1}=U V$

$$
\left\|\alpha_{t}\left(U_{1}\right)-e^{i p t} U_{1}\right\|<\epsilon, t \in[0,1] .
$$

In the same way we have an isometry $U_{2}$ such that $U_{2} U_{2}^{*}=e_{2}$ and

$$
\left\|\alpha_{t}\left(U_{2}\right)-e^{-i p t} U_{2}\right\|<\epsilon, t \in[0,1] .
$$

Let $h_{1}, h_{2}$.be $\mathrm{C}^{\infty}$-functions on $\mathbf{R}$ such that

$$
\begin{aligned}
& 0 \leq h_{1} \leq 1,0 \leq h_{2} \leq 1, \\
& \operatorname{supp} h_{1} \subset[-5 p / 2, p / 2], \operatorname{supp}_{2} \subset[-p / 2,5 p / 2], \\
& h_{1}^{2}+h_{2}^{2}=1 \text { on }[-2 p, 2 p] .
\end{aligned}
$$

Let, as in the proof of Lemma 6 in [22],

$$
x=U_{1} h_{1}(H)+U_{2} h_{2}(H)
$$

where $H$ is the generator of the canonical unitary group $\lambda_{t}$ in the multiplier algebra of $A \times \mathbf{R}$, and

$$
\begin{aligned}
& h(H)=\int \hat{h}(t) \lambda_{t} d t \\
& \hat{h}(t)=\frac{1}{2 \pi} \int e^{-i t q} h(q) d q
\end{aligned}
$$

Then

$$
\begin{aligned}
& x x^{*}=h_{1}^{2}(H)+h_{2}^{2}(H) \\
& x x^{*} \approx h_{1}^{2}(H-p) e_{1}+h_{2}^{2}(H+p) e_{2}+h_{1} h_{2}(H-p) V_{1} V_{2}^{*}+h_{1} h_{2}(H+p) V_{2} V_{1}^{*} .
\end{aligned}
$$

If $f$ is a continuous function on $\mathbf{R}$ such that $f(t)=1$ on $[-3 p / 2,3 p / 2]$ and $\operatorname{supp}(f) \subset$ $[-2 p, 2 p]$, then for $y=f(x)$, it follows that $x^{*} x y=y$ and $\left\|y x x^{*}-x x^{*}\right\|$ is small depending on $\epsilon$. If $\epsilon>0$ is sufficiently small then $\left\|y f_{1 / 8}\left(\left|x^{*}\right|\right)-f_{1 / 8}\left(\left|x^{*}\right|\right)\right\|<1 / 4$, where $f_{s}$ is a continuous function on $\mathbf{R}$ such that $f_{s}(t)=1$ for $t \geq s, f_{s}(t)=0$ for $t \leq s / 2$, and linear on $[s / 2, s]$. Then it follows that $\left\|f_{1 / 2}(|x|) f_{1 / 8}\left(x^{*}\right)-f_{1 / 8}\left(\left|x^{*}\right|\right)\right\|<1 / 4$, i.e, $x$ is an approximate scaling element. By Lemma 4.2 of [1] $A \times \mathbf{R}$ contains an infinite projection. The rest of the proof goes exactly as the proof of Theorem 2 of [22] by using Theorems 4.1 and 2.1 . 


\section{References}

[1] B. Blackadar and J. Cuntz, The structure of stable algebraically simple $C^{*}$-algebras, Amer. J. Math. 104, 813-822 (1982)

[2] O. Bratteli, G.A. Elliott, D.E. Evans, and A. Kishimoto, Homotopy of a pair of approximately commuting unitaries in a simple purely infinite unital $C^{*}$-algebra, preprint.

[3] O. Bratteli, D.E. Evans, and A. Kishimoto, The Rohlin property for quasi-free automorphisms of the Fermion algebra, Proc. London Math. Soc. (to appear)

[4] O. Bratteli, D.E. Evans, and A. Kishimoto, Almost shift invariant projections in infinite tensor products, in Quantum and Non-Commutative Analysis $\mathrm{H}$. Araki et al eds. Kluwer, 427-434, 1993.

[5] O. Bratteli, A. Kishimoto, M. Rørdam, and E. Størmer, The crossed product of a UHF algebra by a shift, Ergod. Th. \& Dynam. Sys. 13, 615-626 (1993)

[6] O. Bratteli and D.W. Robinson, Operator algebras and quantum statistical mechanics I, Springer-Verlag, New York, Heidelberg, Berlin, 1979

[7] L. Brown and G.K. Pedersen, $C^{*}$-algebras of real rank zero, J. Func. Anal. 99, 131$149(1991)$

[8] A. Connes, Outer conjugacy class of automorphisms of factors, Ann. Scient. Ec. Norm. Sup., $4^{e}$ serie, 8, 383-420 (1975)

[9] A. Connes and N. Higson, Deformations, morphismes asymptotiques et K-theorie bivariante, C.R. Acad. Sci. Par. Ser, I Math. 310, 101-106 (1990)

[10] J. Cuntz, Simple $C^{*}$-algebras generated by isometries, Comm. Math. Phys. 57, 173$185(1977)$

[11] J. Cuntz, K-theory for certain $C^{*}$-algebras, Ann. Math. 113, 181-197 (1981)

[12] R.H. Herman and A. Ocneanu, Stability for integer actions on UHF $C^{*}$-algebras, J. Funct. Anal. 59, 132-144 (1984)

[13] R.H. Herman and A. Ocneanu, Spectral Analysis for automorphisms of UHF $C^{*}$ algebras, J. Funct. Anal. 66, 1-10 (1986)

[14] A. Kishimoto, Simple crossed products by locally compact abelian groups, Yokohama Math. J. 28, 69-85 (1980)

[15] A. Kishimoto, Outer automorphisms and reduced crossed products of simple $C^{*}$ algebras, Comm. Math. Phys. 81, 429-435 (1981) 
[16] A. Kishimoto, Automorphisms and covariant irreducible representations, Yokohama Math. J. 31, 159-168 (1983)

[17] A. Kishimoto, Type I orbits in the pure states of a $C^{*}$-dynamical system, I, II, Publ. RIMS, Kyoto Univ. 23, 321-336, 517-526 (1987)

[18] A. Kishimoto, Outer automorphism subgroups of a compact abelian ergodic action, J. Operator Theory 20, 59-67 (1988)

[19] A. Kishimoto, The Rohlin property for shifts on UHF algebras and automorphisms of Cuntz algebras, preprint.

[20] A. Kishimoto, The Rohlin property for automorphisms of UHF algebras, J. reine angew. Math. (to appear)

[21] A. Kishimoto and A. Kumjian, Simple stably projectionless $C^{*}$-algebras arising as crossed products, Canadian J. Math. (to appear)

[22] A. Kishimoto and A. Kumjian, Crossed products of Cuntz algebras by quasi-free automorphisms, Proceedings of the Fields Institute (to appear)

[23] A. Kishimoto and D.W. Robinson, Dissipations, derivations, dynamical systems, and asymptotic abelianess, J. Operator Theory 13, 155-187 (1985)

[24] H. Lin, Exponential rank of $C^{*}$-algebras with real rank zero and the Brown-Pedersen conjecture, J. Funct. Anal. 114, 1-11 (1993)

[25] G.K. Pedersen, $C^{*}$-algebras and their automorphism groups, Academic Press, London, New York, San Francisco, 1979

[26] M. Rørdam, Classification of certain infinite simple $C^{*}$-algebras, J. Funct. Anal. (to appear)

[27] S. Sakai, Operator algebras in dynamical systems, Cambridge Univ. Press, Cambridge, 1991

[28] S. Zhang, A property of purely infinite simple $C^{*}$-algebras, Proc. Amer. math. Soc. (to appear) 